\title{
Infinitely many solutions for equations of $p(x)$-Laplace type with the nonlinear Neumann boundary condition
}

\author{
Eun Bee Choi \\ Department of Mathematical Sciences, Seoul National University, \\ Seoul 151-742, Republic of Korea (eunbee@snu.ac.kr)

\section{Jae-Myoung Kim} \\ Department of Mathematics, Seoul National University, Seoul 151-742, \\ Republic of Korea (cauchy02@naver.com)

\section{Yun-Ho Kim*} \\ Department of Mathematics Education, Sangmyung University, \\ Seoul 110-743, Republic of Korea (kyh1213@smu.ac.kr)
}

(MS received 14 December 2015; accepted 19 April 2016)

We investigate the following nonlinear Neumann boundary-value problem with associated $p(x)$-Laplace-type operator

$$
\left.\begin{array}{rl}
-\operatorname{div}(\varphi(x, \nabla u))+|u|^{p(x)-2} u=f(x, u) & \text { in } \Omega, \\
\varphi(x, \nabla u) \frac{\partial u}{\partial n}=g(x, u) & \text { on } \partial \Omega,
\end{array}\right\}
$$

where the function $\varphi(x, v)$ is of type $|v|^{p(x)-2} v$ with continuous function $p: \bar{\Omega} \rightarrow(1, \infty)$ and both $f: \Omega \times \mathbb{R} \rightarrow \mathbb{R}$ and $g: \partial \Omega \times \mathbb{R} \rightarrow \mathbb{R}$ satisfy a Carathéodory condition. We first show the existence of infinitely many weak solutions for the Neumann problems using the Fountain theorem with the Cerami condition but without the Ambrosetti and Rabinowitz condition. Next, we give a result on the existence of a sequence of weak solutions for problem (P) converging to 0 in $L^{\infty}$-norm by employing De Giorgi's iteration and the localization method under suitable conditions.

Keywords: $p(x)$-Laplace type; Fountain theorem; De Giorgi's iteration; localization method

2010 Mathematics subject classification: Primary 35D30; 35J20; 35J60; 35J92; 46E35

\section{Introduction}

The interest in variational problems with $p(x)$-growth conditions is founded on their prevalence throughout various areas of mathematical physics such as elastic mechanics, electro-rheological fluid dynamics and image processing, etc.; we refer the reader to $[10,29,33]$ and references therein.

* Corresponding author.

(C) 2017 The Royal Society of Edinburgh 
In this paper we are concerned with the existence of infinitely many weak solutions for the following nonlinear Neumann boundary-value problem with associated $p(x)$-Laplace-type operator

$$
\left.\begin{array}{rl}
-\operatorname{div}(\varphi(x, \nabla u))+|u|^{p(x)-2} u=f(x, u) & \text { in } \Omega, \\
\varphi(x, \nabla u) \frac{\partial u}{\partial n}=g(x, u) & \text { on } \partial \Omega,
\end{array}\right\}
$$

where the function $\varphi(x, v)$ is of type $|v|^{p(x)-2} v$ with continuous function $p: \bar{\Omega} \rightarrow$ $(1, \infty), \Omega$ is a bounded domain in $\mathbb{R}^{N}$ for $N \geqslant 3$ with Lipschitz boundary $\partial \Omega, \partial u / \partial n$ denotes the outer normal derivative of $u$ with respect to $\partial \Omega$, and both $f: \Omega \times \mathbb{R} \rightarrow$ $\mathbb{R}$ and $g: \partial \Omega \times \mathbb{R} \rightarrow \mathbb{R}$ satisfy a Carathéodory condition. The $p(x)$-Laplace-type operator $\operatorname{div}(\varphi(x, \nabla u))$, which is the natural generalization of the $p(x)$-Laplace operator $\operatorname{div}\left(|\nabla u|^{p(x)-2} \nabla u\right)$, has been widely studied by many researchers; see $[6$, 18,23-25,29,32] and references therein. Concerning elliptic equations with nonlinear boundary conditions, we refer the reader to $[6,8,28,38,40,41]$.

Superlinear problems have been studied extensively by many authors; see, for instance, $[6,15,23,28,38,40,41]$. In particular, Yao [40] showed the existence of nontrivial solutions for the inhomogeneous and nonlinear Neumann boundary-value problem involving the $p(x)$-Laplacian; see [6] for the $p(x)$-Laplace type. A common feature of these results is the following Ambrosetti and Rabinowitz (AR) condition, which was introduced by Ambrosetti and Rabinowitz [1] for the case in which $p(x) \equiv 2$.

(AR) There exist positive constants $M$ and $\theta$ such that $\theta>p_{+}$and

$$
0<\theta F(x, t) \leqslant f(x, t) t \quad \text { for } x \in \Omega \quad \text { and } \quad|t| \geqslant M
$$

where $p_{+}=\sup _{x \in \Omega} p(x)$ and $F(x, t)=\int_{0}^{t} f(x, s) \mathrm{d} s$.

This condition guarantees the boundedness of the Palais-Smale (PS) sequence of the Euler-Lagrange functional, which plays a crucial role in the applications of critical-point theory. However, the AR condition is quite restrictive because there are many superlinear functions that do not satisfy it. In this direction, Miyagaki and Souto [30] have tried to drop the AR condition for the $p(x) \equiv 2$ case in order to get existence of a non-trivial solution for a superlinear eigenvalue Dirichlet problem by assuming the following condition.

(f1) There exists $t_{0}>0$ such that

$$
\frac{f(x, t)}{t^{p_{+}-1}} \text { is increasing in } t \geqslant t_{0} \text { and decreasing in } t \leqslant-t_{0}
$$

for all $x \in \Omega$, where $\Omega$ is a bounded domain in $\mathbb{R}^{N}$.

Recently, some authors $[7,9,18,22,26]$ generalized the results in [30]. In the case of variable exponents, Ji [22] generalized results in [30] to construct the existence of a non-trivial solution for the $p(x)$-Laplacian Dirichlet problem under the following condition. 
(f2) There is a constant $C_{*}>0$ such that

$$
t f(x, t)-p_{+} F(x, t) \leqslant s f(x, s)-p_{+} F(x, s)+C_{*}
$$

for any $x \in \Omega, 0<t<s$ or $s<t<0$.

Condition ( $f 2)$ was first considered by Miyagaki and Souto [30] in the $p(x) \equiv$ 2 case and it is a weaker condition than $(f 1)$. Also, under condition ( $f 2)$, Ge [18] established the existence of a non-trivial weak solution for the $p(x)$-Laplacian problem involving a non-local term, and, for the case in which $p(x) \equiv p$, Li and Yang [26] proved the existence of a non-trivial weak solution for the $p$-Laplacian problem. Following the basic ideas of [26], Chung and Toan [9] considered a class of nonlinear and non-homogeneous problems in an Orlicz-Sobolev space setting. Recently, using an abstract result contained in [5], the authors in $[2,3]$ obtained the existence of a non-trivial weak solution for a parametric Neumann problem driven by the $p(x)$-Laplacian without the AR condition.

The aims of this paper are twofold. The first is to show the existence of infinitely many weak solutions for problem $(\mathrm{P})$ without the AR condition (see theorem 3.11). Such a result for the elliptic boundary-value problem with nonlinear Neumann boundary condition involving the $p(x)$-Laplacian is very rare even if $p(x)$ is a constant. To the best of our knowledge, most of the results about the existence of weak solutions for Neumann problems are derived under the AR condition; see $[6,8,28,38,40,41]$. Inspired by the papers $[3,18,22,26,30]$, we demonstrate our result in a more general setting.

The second aim is to extend the recent results in $[31,34,36]$, namely, we prove the existence of non-trivial weak solutions for problem $(\mathrm{P})$, the nonlinear Neumann boundary-value problem with associated $p(x)$-Laplacian-type operator (see theorem 4.7). Roughly speaking, we establish the existence of small solutions in the sense that the sequence of solutions converging to 0 in the $L^{\infty}$-norm relies only on local behaviour of the nonlinear equation, under assumptions on $f(x, t)$, only for sufficiently small $t$. Our method is based on employing the global variational formulation-type method and the modified functional method that were first introduced by Wang in [36]. More specifically, the strategy of the modified functional method is to modify and extend $f(x, t)$ to an appropriate $\tilde{f}(x, t)$; using the associated modified functional $\tilde{I}$ with this $\tilde{f}(x, t)$, Wang showed the existence of a sequence of solutions converging to 0 in $L^{\infty}$-norm (see [36] for Neumann problems). Tan and Fang [34] showed this result for the $p(x)$-Laplacian Dirichlet problem using a regularity result of Fan [13] without its proof. Recently, Ho and Sim [21] extended the regularity-type lemma in Vergara and Zacher [35] or Winkert and Zacher [39] and applied such a result to show the boundedness of solutions for the Dirichlet problem with the variable exponents via De Giorgi's technique. Borrowing this idea, we give a direct proof of the $L^{\infty}$-bound of weak solutions for problem $(\mathrm{P})$, modifying that of [20, theorem 4.2]. To put it briefly, the following two aspects are worth mentioning. First, we show the existence of infinitely many weak solutions for the Neumann problems (P) using the Fountain theorem with the Cerami condition under a more complicated nonlinear boundary condition in comparison to [20]. Second, we establish the existence of a sequence of weak solutions for problems converging to 0 in $L^{\infty}$-norm based on the regularity lemma in [21], which is slightly different from that of [39, theorem 1.1]. 
This paper is organized as follows. In $\S 2$ we recall some basic results for the variable exponent Lebesgue-Sobolev spaces. In $\S 3$, under certain conditions on $\varphi$, $f$ and $g$, we establish the existence result of infinitely many weak solutions for problem $(\mathrm{P})$ (theorem 3.11) by employing as the main tool the variational principle. In $\S 4$ we prove the existence of infinitely many weak solutions (theorem 4.7) for the boundary-value problem of nonlinear type based on the global variational formulations-type method and the modified functional method.

\section{Preliminaries}

In this section we recall some definitions and basic properties of the variable exponent Lebesgue spaces $L^{p(\cdot)}(\Omega)$ and the variable exponent Lebesgue-Sobolev spaces $W^{1, p(\cdot)}(\Omega)$ that will be treated in the next sections. For a deeper treatment on these spaces, we refer the reader to $[10,11,16,23]$.

Set

$$
C_{+}(\bar{\Omega})=\left\{h \in C(\bar{\Omega}): \min _{x \in \bar{\Omega}} h(x)>1\right\} .
$$

For any $h \in C_{+}(\bar{\Omega})$, we define

$$
h_{+}=\sup _{x \in \Omega} h(x) \quad \text { and } \quad h_{-}=\inf _{x \in \Omega} h(x) .
$$

For any $p \in C_{+}(\bar{\Omega})$, we introduce the variable exponent Lebesgue space

$$
L^{p(\cdot)}(\Omega):=\left\{u: u \text { is a measurable real-valued function, } \int_{\Omega}|u(x)|^{p(x)} \mathrm{d} x<\infty\right\},
$$

endowed with the Luxemburg norm

$$
\|u\|_{L^{p(\cdot)}(\Omega)}=\inf \left\{\lambda>0: \int_{\Omega}\left|\frac{u(x)}{\lambda}\right|^{p(x)} \mathrm{d} x \leqslant 1\right\} .
$$

The dual space of $L^{p(\cdot)}(\Omega)$ is $L^{p^{\prime}(\cdot)}(\Omega)$, where $1 / p(x)+1 / p^{\prime}(x)=1$.

The variable exponent Sobolev space $W^{1, p(\cdot)}(\Omega)$ is defined by

$$
W^{1, p(\cdot)}(\Omega)=\left\{u \in L^{p(\cdot)}(\Omega):|\nabla u| \in L^{p(\cdot)}(\Omega)\right\},
$$

where the norm is

$$
\|u\|_{W^{1, p(\cdot)}(\Omega)}=\|u\|_{L^{p(\cdot)}(\Omega)}+\|\nabla u\|_{L^{p(\cdot)}(\Omega)} .
$$

Next, we recall elementary inequalities below.

LEMMA 2.1 (Fan and Zhao [16]). The space $L^{p(\cdot)}(\Omega)$ is a separable, uniformly convex Banach space and its conjugate space is $L^{p^{\prime}(\cdot)}(\Omega)$, where $1 / p(x)+1 / p^{\prime}(x)=1$. For any $u \in L^{p(\cdot)}(\Omega)$ and $v \in L^{p^{\prime}(\cdot)}(\Omega)$, we have

$$
\left|\int_{\Omega} u v \mathrm{~d} x\right| \leqslant\left(\frac{1}{p_{-}}+\frac{1}{\left(p^{\prime}\right)_{-}}\right)\|u\|_{L^{p(\cdot)}(\Omega)}\|v\|_{L^{p^{\prime}(\cdot)}(\Omega)} \leqslant 2\|u\|_{L^{p(\cdot)}(\Omega)}\|v\|_{L^{p^{\prime}(\cdot)}(\Omega)} .
$$


Lemma 2.2 (Fan and Zhao [16]). Define

$$
\rho(u)=\int_{\Omega}|u|^{p(x)} \mathrm{d} x \quad \text { for all } u \in L^{p(\cdot)}(\Omega) .
$$

Then

(1) $\rho(u)>1$ (respectively, $\rho(u)=1, \rho(u)<1)$ if and only if $\|u\|_{L^{p(\cdot)}(\Omega)}>1$ (respectively, $\left.\|u\|_{L^{p(\cdot)}(\Omega)}=1,\|u\|_{L^{p(\cdot)}(\Omega)}<1\right)$;

(2) if $\|u\|_{L^{p(\cdot)(\Omega)}}>1$, then $\|u\|_{L^{p(\cdot)}(\Omega)}^{p_{-}} \leqslant \rho(u) \leqslant\|u\|_{L^{p(\cdot)}(\Omega)}^{p_{+}}$;

(3) if $\|u\|_{L^{p(\cdot)}(\Omega)}<1$, then $\|u\|_{L^{p(\cdot)}(\Omega)}^{p_{+}} \leqslant \rho(u) \leqslant\|u\|_{L^{p(\cdot)}(\Omega)}^{p_{-}}$.

Similarly, we deduce the following lemma.

Lemma 2.3. If we define

$$
\rho_{1}(u)=\int_{\Omega}|u|^{p(x)}+|\nabla u|^{p(x)} \mathrm{d} x \quad \text { for all } u \in W^{1, p(\cdot)}(\Omega),
$$

then

(1) $\rho_{1}(u)>1$ (respectively, $\left.\rho_{1}(u)=1, \rho_{1}(u)<1\right)$ if and only if $\|u\|_{W^{1, p(\cdot)}(\Omega)}>1$ (respectively, $\left.\|u\|_{W^{1, p(\cdot)}(\Omega)}=1,\|u\|_{W^{1, p(\cdot)}(\Omega)}<1\right)$;

(2) if $\|u\|_{W^{1, p(\cdot)}(\Omega)}>1$, then $\|u\|_{W^{1, p(\cdot)}(\Omega)}^{p_{-}} \leqslant \rho_{1}(u) \leqslant\|u\|_{W^{1, p(\cdot)}(\Omega)}^{p_{+}}$;

(3) if $\|u\|_{W^{1, p(\cdot)}(\Omega)}<1$, then $\|u\|_{W^{1, p(\cdot)}(\Omega)}^{p_{+}} \leqslant \rho_{1}(u) \leqslant\|u\|_{W^{1, p(\cdot)}(\Omega)}^{p_{-}}$.

LEMma 2.4 (Fan and Zhao [16]). Let $\Omega \subset \mathbb{R}^{N}$ be an open, bounded set with Lipschitz boundary and let $p \in C_{+}(\bar{\Omega})$ with $1<p_{-} \leqslant p_{+}<\infty$. If $q \in L^{\infty}(\Omega)$ with $q_{-}>1$ satisfies

$$
q(x) \leqslant p^{*}(x):= \begin{cases}\frac{N p(x)}{N-p(x)} & \text { if } N>p(x) \\ +\infty & \text { if } N \leqslant p(x)\end{cases}
$$

for all $x \in \Omega$, then we have

$$
W^{1, p(\cdot)}(\Omega) \hookrightarrow L^{q(\cdot)}(\Omega)
$$

and the embedding is compact if $\inf _{x \in \Omega}\left(p^{*}(x)-q(x)\right)>0$.

Lemma 2.5 (Fan [14]). Let $\Omega \subset \mathbb{R}^{N}, N \geqslant 2$, be a bounded domain with smooth boundary. Suppose that $p \in C_{+}(\bar{\Omega})$ and $r \in C(\partial \Omega)$ satisfy the condition

$$
1 \leqslant r(x)<p^{\partial}(x):= \begin{cases}\frac{(N-1) p(x)}{N-p(x)} & \text { if } N>p(x), \\ +\infty & \text { if } N \leqslant p(x)\end{cases}
$$

for all $x \in \partial \Omega$. Then the embedding $W^{1, p(\cdot)}(\Omega) \hookrightarrow L^{r(\cdot)}(\partial \Omega)$ is compact and continuous.

Throughout this paper, we write $X:=W^{1, p(\cdot)}(\Omega)$ and $X^{*}$ is the dual space of $X$. Furthermore, $\langle\cdot, \cdot\rangle$ denotes the pairing of $X$ and its dual $X^{*}$. 


\section{Existence of infinitely many weak solutions}

In this section we show the existence of infinitely many solutions for problem (P) by applying the Fountain theorem under the Cerami condition.

Definition 3.1. We say that $u \in X$ is a weak solution of problem (P) if

$$
\int_{\Omega} \varphi(x, \nabla u) \cdot \nabla v \mathrm{~d} x+\int_{\Omega}|u|^{p(x)-2} u v \mathrm{~d} x=\int_{\Omega} f(x, u) v \mathrm{~d} x+\int_{\partial \Omega} g(x, u) v \mathrm{~d} S
$$

for all $v \in X$.

Suppose that $\varphi: \Omega \times \mathbb{R}^{N} \rightarrow \mathbb{R}^{N}$ is the continuous derivative with respect to $v$ of the mapping $\Phi_{0}: \Omega \times \mathbb{R}^{N} \rightarrow \mathbb{R}, \Phi_{0}=\Phi_{0}(x, v)$, namely, $\varphi(x, v)=(\mathrm{d} / \mathrm{d} v) \Phi_{0}(x, v)$. We assume that $\varphi$ and $\Phi_{0}$ satisfy the following assumptions.

(J1) The equality

$$
\Phi_{0}(x, \mathbf{0})=0
$$

holds for almost all $x \in \Omega$.

(J2) There is a function $a \in L^{p^{\prime}(\cdot)}(\Omega)$ and a non-negative constant $b$ such that

$$
|\varphi(x, v)| \leqslant a(x)+b|v|^{p(x)-1}
$$

for almost all $x \in \Omega$ and for all $v \in \mathbb{R}^{N}$.

(J3) $\Phi_{0}(x, \cdot)$ is strictly convex in $\mathbb{R}^{N}$ for all $x \in \Omega$.

(J4) There exists a positive constant $d$ such that

$$
d|v|^{p(x)} \leqslant \varphi(x, v) \cdot v \quad \text { and } \quad d|v|^{p(x)} \leqslant p_{+} \Phi_{0}(x, v)
$$

for all $x \in \Omega$ and $v \in \mathbb{R}^{N}$.

(J5) There exists a positive constant $\mu_{1}$ such that

$$
\mathcal{H}(x, s v) \leqslant \mathcal{H}(x, v)+\mu_{1}
$$

for $v \in \mathbb{R}^{N}$ and $s \in[0,1]$, where $\mathcal{H}(x, v)=p_{+} \Phi_{0}(x, v)-\varphi(x, v) \cdot v$ for almost all $x \in \Omega$.

Let us define the functional $\Phi: X \rightarrow \mathbb{R}$ by

$$
\Phi(u)=\int_{\Omega} \Phi_{0}(x, \nabla u) \mathrm{d} x+\int_{\Omega} \frac{1}{p(x)}|u|^{p(x)} \mathrm{d} x .
$$

Under assumptions (J1), (J2) and (J4), it follows from [23,29] that the functional $\Phi$ is well defined on $X, \Phi \in C^{1}(X, \mathbb{R})$ and its Fréchet derivative is given by

$$
\left\langle\Phi^{\prime}(u), v\right\rangle=\int_{\Omega} \varphi(x, \nabla u) \cdot \nabla v \mathrm{~d} x+\int_{\Omega}|u|^{p(x)-2} u v \mathrm{~d} x .
$$

We give some examples that satisfy assumption (J5). 
EXAMPle 3.2. Let us consider

$$
\varphi(x, t)=|t|^{p(x)-2} t \quad \text { and } \quad \Phi_{0}(x, t)=\frac{1}{p(x)}|t|^{p(x)}
$$

for $t \in \mathbb{R}$. Then $\mathcal{H}(x, s t) \leqslant \mathcal{H}(x, t)$ for all $s \in[0,1]$, and so assumption (J5) holds for any positive constant $\mu_{1}$.

EXAMPle 3.3. Let us consider

$$
\varphi(x, t)=\left(1+t^{2}\right)^{(p(x)-2) / 2} t \quad \text { and } \quad \Phi_{0}(x, t)=\frac{1}{p(x)}\left[\left(1+t^{2}\right)^{p(x) / 2}-1\right]
$$

for $t \in \mathbb{R}$, where $p(x) \geqslant 2$ for all $x \in \bar{\Omega}$. Then

$$
\mathcal{H}(x, t)=p_{+} \Phi_{0}(x, t)-\varphi(x, t) t=\frac{p_{+}}{p(x)}\left[\left(1+t^{2}\right)^{p(x) / 2}-1\right]-\left(1+t^{2}\right)^{(p(x)-2) / 2} t^{2} \geqslant 0
$$

for any $t \in \mathbb{R}$. For fixed $t$, the function $\mathcal{H}(x, s t)$ is continuous for $s \in[0,1]$. Thus, we can choose $s_{0} \in[0,1]$ such that $\mathcal{H}\left(x, s_{0} t\right)=\max _{s \in[0,1]} \mathcal{H}(x, s t)$. It is obvious that $s_{0}>0$. If $s_{0}=1$, then $\mathcal{H}(x, s t) \leqslant \mathcal{H}(x, t)$ for all $s \in[0,1]$ and $t \in \mathbb{R}$. If $0<s_{0}<1$, we have

$$
\lim _{|t| \rightarrow \infty} \frac{\mathcal{H}\left(x, s_{0} t\right)}{\mathcal{H}(x, t)}=s_{0}^{p(x)}<1 .
$$

Therefore, there exists $t_{0}$ large enough such that $\mathcal{H}\left(x, s_{0} t\right) / \mathcal{H}(x, t)<1$ for all $|t|>t_{0}$ and so $\mathcal{H}\left(x, s_{0} t\right)<\mathcal{H}(x, t)$ for all $|t|>t_{0}$. Put

$$
\mu_{1}:=1+\max _{(t, s) \in\left[-t_{0}, t_{0}\right] \times[0,1]} \mathcal{H}(x, s t) .
$$

It follows that $\mathcal{H}(x, s t)<\mathcal{H}(x, t)+\mu_{1}$ for all $t \in \mathbb{R}$ and $s \in[0,1]$, that is, condition (J5) holds.

EXAmPle 3.4. Let us consider

$$
\varphi(x, v)=\left(1+\frac{|v|^{p(x)}}{\sqrt{1+|v|^{2 p(x)}}}\right)|v|^{p(x)-2} v
$$

and

$$
\Phi_{0}(x, v)=\frac{1}{p(x)}\left(|v|^{p(x)}+\sqrt{1+|v|^{2 p(x)}}-1\right)
$$

for $v \in \mathbb{R}^{N}$. Then

$$
\begin{aligned}
\mathcal{H}(x, v) & =p_{+} \Phi_{0}(x, v)-\varphi(x, v) \cdot v \\
& =\frac{p_{+}}{p(x)}\left(|v|^{p(x)}+\sqrt{1+|v|^{2 p(x)}}-1\right)-\left(1+\frac{|v|^{p(x)}}{\sqrt{1+|v|^{2 p(x)}}}\right)|v|^{p(x)}
\end{aligned}
$$

for any $v \in \mathbb{R}^{N}$. It is easy to show that

$$
\mathcal{H}(x, v)-\mathcal{H}(x, s v) \geqslant-1 .
$$

Thus, condition (J5) holds for $\mu_{1}>1$. 
The following assertion can be found in [24].

Lemma 3.5. Assume that (J1)-(J4) hold. Then the functional $\Phi: X \rightarrow \mathbb{R}$ is convex and weakly lower semi-continuous on $X$. Moreover, the operator $\Phi^{\prime}$ is a mapping of type $\left(S_{+}\right)$, i.e. if $u_{n} \rightarrow u$ in $X$ and $\lim \sup _{n \rightarrow \infty}\left\langle\Phi^{\prime}\left(u_{n}\right)-\Phi^{\prime}(u), u_{n}-u\right\rangle \leqslant 0$, then $u_{n} \rightarrow u$ in $X$ as $n \rightarrow \infty$.

Next we need the following assumptions for $f$ and $g$. Defining

$$
F(x, t)=\int_{0}^{t} f(x, s) \mathrm{d} s \text { and } G(x, t)=\int_{0}^{t} g(x, s) \mathrm{d} s
$$

we then assume that the following hold.

(F1) $f: \Omega \times \mathbb{R} \rightarrow \mathbb{R}$ satisfies the Carathéodory condition in the sense that $f(\cdot, t)$ is measurable for all $t \in \mathbb{R}$ and $f(x, \cdot)$ is continuous for almost all $x \in \Omega$.

(F2) $f: \bar{\Omega} \times \mathbb{R} \rightarrow \mathbb{R}$ is a continuous function and there exist two constants $d_{1} \geqslant 0$ and $d_{2}>0$ such that

$$
|f(x, t)| \leqslant d_{1}+d_{2}|t|^{\alpha(x)-1}
$$

for all $x \in \Omega$ and for all $t \in \mathbb{R}$, where $\alpha \in C_{+}(\bar{\Omega})$ and $p_{+}<\alpha(x)<p^{*}(x)$ for all $x \in \bar{\Omega}$.

(F3) $\lim _{|t| \rightarrow+\infty}\left(F(x, t) /|t|^{p_{+}}\right)=+\infty$ uniformly for all $x \in \Omega$.

(F4) There exists a positive constant $\mu_{2}$ such that

$$
\mathcal{F}(x, t) \leqslant \mathcal{F}(x, s)+\mu_{2}
$$

for any $x \in \Omega, 0<t<s$ or $s<t<0$, where $\mathcal{F}(x, t)=t f(x, t)-p_{+} F(x, t)$.

(F5) $f(x,-t)=-f(x, t)$ holds for all $(x, t) \in \Omega \times \mathbb{R}$.

(G1) $g: \partial \Omega \times \mathbb{R} \rightarrow \mathbb{R}$ satisfies the Carathéodory condition and there exist two constants $d_{3} \geqslant 0$ and $d_{4}>0$ such that

$$
|g(x, t)| \leqslant d_{3}+d_{4}|t|^{\beta(x)-1}
$$

for all $x \in \partial \Omega$ and for all $t \in \mathbb{R}$, where $\beta \in C_{+}(\partial \Omega)$ and $p_{+}<\beta(x)<p^{\partial}(x)$ for all $x \in \partial \Omega$.

(G2) $\lim _{|t| \rightarrow+\infty}\left(G(x, t) /|t|^{p_{+}}\right)=+\infty$ uniformly for all $x \in \partial \Omega$.

(G3) There exists a positive constant $\mu_{3}$ such that

$$
\mathcal{G}(x, t) \leqslant \mathcal{G}(x, s)+\mu_{3}
$$

for any $x \in \partial \Omega, 0<t<s$ or $s<t<0$, where $\mathcal{G}(x, t)=\operatorname{tg}(x, t)-p_{+} G(x, t)$.

(G4) $g(x,-t)=-g(x, t)$ holds for all $(x, t) \in \partial \Omega \times \mathbb{R}$. 
Define the functionals $\Psi, J: X \rightarrow \mathbb{R}$ by

$$
\Psi(u)=\int_{\Omega} F(x, u) \mathrm{d} x \quad \text { and } \quad J(u)=\int_{\partial \Omega} G(x, u) \mathrm{d} S .
$$

Then it is easy to check that $\Psi, J \in C^{1}(X, \mathbb{R})$ and their Fréchet derivatives are

$$
\left\langle\Psi^{\prime}(u), v\right\rangle=\int_{\Omega} f(x, u) v \mathrm{~d} x \quad \text { and } \quad\left\langle J^{\prime}(u), v\right\rangle=\int_{\partial \Omega} g(x, u) v \mathrm{~d} S
$$

for any $u, v \in X$. Define the functional $I: X \rightarrow \mathbb{R}$ by

$$
I(u)=\Phi(u)-\Psi(u)-J(u)
$$

Then it follows that the functional $I \in C^{1}(X, \mathbb{R})$ and its Fréchet derivative is

$$
\begin{aligned}
\left\langle I^{\prime}(u), v\right\rangle= & \int_{\Omega} \varphi(x, \nabla u) \cdot \nabla v \mathrm{~d} x+\int_{\Omega}|u|^{p(x)-2} u v \mathrm{~d} x-\int_{\Omega} f(x, u) v \mathrm{~d} x \\
& -\int_{\partial \Omega} g(x, u) v \mathrm{~d} S
\end{aligned}
$$

for any $u, v \in X$.

Lemma 3.6. Assume that (F1), (F2) and (G1) hold. Then $\Psi$ and $J$ are weaklystrongly continuous on $X$ and their derivative operators are compact.

Proof. Proceeding with an argument analogous to that of [6, proposition 3], it follows that functionals $\Psi$ and $J$ are weakly-strongly continuous on $X$; see also [40].

For $c \in \mathbb{R}$, we say that the energy functional $I$ satisfies the Cerami condi-

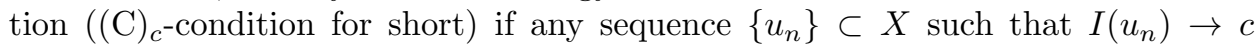
and $\left\|I^{\prime}\left(u_{n}\right)\right\|_{X^{*}}\left(1+\left\|u_{n}\right\|_{X}\right) \rightarrow 0$ as $n \rightarrow \infty$ has a convergent subsequence; such a sequence is then called a Cerami sequence, or a $(\mathrm{C})_{c}$-sequence for short. We next show that the energy functional $I$ satisfies the $(\mathrm{C})_{c}$-condition, which plays a key role in obtaining our main result in this section.

REMARK 3.7. One of the key assumptions for proving that the functional $I$ satisfies the $(\mathrm{C})_{c}$-condition (or the (PS)-condition) for $c \in \mathbb{R}$ is that

$$
f(x, t)=o\left(|t|^{p_{+}-1}\right) \quad \text { as }|t| \rightarrow 0 \text { uniformly for } x \in \Omega
$$

and

$$
g(x, t)=o\left(|t|^{p_{+}-1}\right) \quad \text { as }|t| \rightarrow 0 \text { uniformly for } x \in \partial \Omega
$$

see, for instance, $[4,8,22,27,30,38,40]$. However, we prove the following result without assumptions (3.3) and (3.4).

Lemma 3.8. Assume that (J1)-(J5), (F1)-(F4) and (G1)-(G3) hold. Then the energy functional $I$ satisfies the $(\mathrm{C})_{c}$-condition. 
Proof. Given $c \in \mathbb{R}$, let $\left\{u_{n}\right\} \subset X$ be a $(\mathrm{C})_{c}$-sequence of the functional $I$, that is,

$$
I\left(u_{n}\right) \rightarrow c \text { and }\left\|I^{\prime}\left(u_{n}\right)\right\|_{X^{*}}\left(1+\left\|u_{n}\right\|_{X}\right) \rightarrow 0 \quad \text { as } n \rightarrow \infty,
$$

which shows that

$$
c=I\left(u_{n}\right)+o(1) \quad \text { and } \quad\left\langle I^{\prime}\left(u_{n}\right), u_{n}\right\rangle=o(1),
$$

where $o(1) \rightarrow 0$ as $n \rightarrow \infty$. Note that $\Psi^{\prime}$ and $J^{\prime}$ are mappings of type $\left(S_{+}\right)$by lemma 3.6. Since $I^{\prime}$ is a mapping of type $\left(S_{+}\right)$and $X$ is reflexive by lemmas 3.5 and 2.1 , respectively, it suffices to show the boundedness of the sequence $\left\{u_{n}\right\}$ in $X$. If $\left\{u_{n}\right\}$ is unbounded in $X$, then we can assume that $\left\|u_{n}\right\|_{X}>1$ and $\left\|u_{n}\right\|_{X} \rightarrow \infty$ as $n \rightarrow \infty$. Define a sequence $\left\{w_{n}\right\}$ in $X$ with $w_{n}=u_{n} /\left\|u_{n}\right\|_{X}$. Then $\left\|w_{n}\right\|_{X}=1$ for all $n \in \mathbb{N}$. Therefore, by passing to a subsequence, still denoted by $\left\{w_{n}\right\}$, we have that $\left\{w_{n}\right\}$ converges weakly to $w \in X$ and

$$
\begin{aligned}
& w_{n}(x) \rightarrow w(x) \quad \text { a.e. in } \Omega \quad \text { and } \quad w_{n} \rightarrow w \quad \text { in } L^{\alpha(\cdot)}(\Omega) \quad \text { as } n \rightarrow \infty \text {, } \\
& w_{n}(x) \rightarrow w(x) \text { a.e. in } \partial \Omega \text { and } \quad w_{n} \rightarrow w \quad \text { in } L^{\beta(\cdot)}(\partial \Omega) \quad \text { as } n \rightarrow \infty \text {, }
\end{aligned}
$$

where 'a.e.' indicates 'almost everywhere', using lemmas 2.4 and 2.5. Let $\Omega_{0}=$ $\{x \in \bar{\Omega}: w(x) \neq 0\}$. From (3.6), $\left|u_{n}(x)\right|=\left|w_{n}(x)\right|\left\|u_{n}\right\|_{X} \rightarrow+\infty$ as $n \rightarrow \infty$ for $x \in \Omega_{0} \cap \Omega$. Similarly, due to (3.7), we know that $\left|u_{n}(x)\right| \rightarrow+\infty$ as $n \rightarrow \infty$ for $x \in \Omega_{0} \cap \partial \Omega$. It follows from (3.5), (J4) and lemma 2.3 that

$$
\begin{aligned}
c=I\left(u_{n}\right)+o(1)= & \int_{\Omega} \Phi_{0}\left(x, \nabla u_{n}\right) \mathrm{d} x+\int_{\Omega} \frac{1}{p(x)}\left|u_{n}\right|^{p(x)} \mathrm{d} x-\int_{\Omega} F\left(x, u_{n}\right) \mathrm{d} x \\
& \quad-\int_{\partial \Omega} G\left(x, u_{n}\right) \mathrm{d} S+o(1) \\
\geqslant & C_{1}\left\|u_{n}\right\|_{X}^{p_{-}}-\int_{\Omega} F\left(x, u_{n}\right) \mathrm{d} x-\int_{\partial \Omega} G\left(x, u_{n}\right) \mathrm{d} S+o(1)
\end{aligned}
$$

for some constant $C_{1}$, and thus

$$
\int_{\Omega} F\left(x, u_{n}\right) \mathrm{d} x+\int_{\partial \Omega} G\left(x, u_{n}\right) \mathrm{d} S \geqslant C_{1}\left\|u_{n}\right\|_{X}^{p_{-}}-c+o(1) \rightarrow+\infty \quad \text { as } n \rightarrow \infty .
$$

Also, by assumption (J2) and lemmas 2.1 and 2.3 , we get

$$
\begin{aligned}
I\left(u_{n}\right)= & \int_{\Omega} \Phi_{0}\left(x, \nabla u_{n}\right) \mathrm{d} x+\int_{\Omega} \frac{1}{p(x)}\left|u_{n}\right|^{p(x)} \mathrm{d} x-\int_{\Omega} F\left(x, u_{n}\right) \mathrm{d} x \\
& -\int_{\partial \Omega} G\left(x, u_{n}\right) \mathrm{d} S \\
\leqslant & \int_{\Omega} a(x)\left|\nabla u_{n}\right| \mathrm{d} x+\frac{b}{p_{-}} \int_{\Omega}\left|\nabla u_{n}\right|^{p(x)} \mathrm{d} x+\frac{1}{p_{-}} \int_{\Omega}\left|u_{n}\right|^{p(x)} \mathrm{d} x \\
& \quad-\int_{\Omega} F\left(x, u_{n}\right) \mathrm{d} x-\int_{\partial \Omega} G\left(x, u_{n}\right) \mathrm{d} S \\
\leqslant & \left(2\|a\|_{L^{p^{\prime}(\cdot)(\Omega)}}+b+1\right)\left\|u_{n}\right\|_{X}^{p_{+}}-\int_{\Omega} F\left(x, u_{n}\right) \mathrm{d} x-\int_{\partial \Omega} G\left(x, u_{n}\right) \mathrm{d} S,
\end{aligned}
$$


and we deduce from (3.5) that

$$
\frac{2\|a\|_{L^{p^{\prime}(\cdot)}(\Omega)}+b+1}{\int_{\Omega} F\left(x, u_{n}\right) \mathrm{d} x+\int_{\partial \Omega} G\left(x, u_{n}\right) \mathrm{d} S+c-o(1)} \geqslant \frac{1}{\left\|u_{n}\right\|_{X}^{p_{+}}}
$$

for $n$ large enough. In addition, condition (F3) implies that there exists $t_{0}>1$ such that $F(x, t)>|t|^{p_{+}}$for all $x \in \Omega$ and $|t|>t_{0}$. Since $F(x, t)$ is continuous on $\bar{\Omega} \times\left[-t_{0}, t_{0}\right]$ by (F2), there exists a positive constant $C_{2}$ such that $|F(x, t)| \leqslant C_{2}$ for all $(x, t) \in \bar{\Omega} \times\left[-t_{0}, t_{0}\right]$. Therefore, we can choose $C_{3} \in \mathbb{R}$ such that $F(x, t) \geqslant C_{3}$ for all $(x, t) \in \bar{\Omega} \times \mathbb{R}$, and thus

$$
\frac{F\left(x, u_{n}(x)\right)-C_{3}}{\left\|u_{n}\right\|_{X}^{p_{+}}} \geqslant 0
$$

for all $x \in \Omega$ and for all $n \in \mathbb{N}$. Similarly, using assumption (G2), we see that there exists $C_{4} \in \mathbb{R}$ such that

$$
\frac{G\left(x, u_{n}(x)\right)-C_{4}}{\left\|u_{n}\right\|_{X}^{p_{+}}} \geqslant 0
$$

for all $x \in \partial \Omega$ and for all $n \in \mathbb{N}$. Observe that assumptions (F3) and (G2) imply that

$$
\lim _{n \rightarrow \infty} \frac{F\left(x, u_{n}(x)\right)}{\left\|u_{n}\right\|_{X}^{p_{+}}}=\lim _{n \rightarrow \infty} \frac{F\left(x, u_{n}(x)\right)}{\left|u_{n}(x)\right|^{p_{+}}}\left|w_{n}(x)\right|^{p_{+}}=+\infty, \quad x \in \Omega \cap \Omega_{0},
$$

and

$$
\lim _{n \rightarrow \infty} \frac{G\left(x, u_{n}(x)\right)}{\left\|u_{n}\right\|_{X}^{p_{+}}}=\lim _{n \rightarrow \infty} \frac{G\left(x, u_{n}(x)\right)}{\left|u_{n}(x)\right|^{p_{+}}}\left|w_{n}(x)\right|^{p_{+}}=+\infty, \quad x \in \partial \Omega \cap \Omega_{0} .
$$

Now, we claim that $\left|\Omega_{0}\right|=0$, where $|\Omega|$ denotes the Lebesque measure of $\Omega$. If $\left|\Omega_{0}\right| \neq 0$, then by (3.8)-(3.13) and Fatou's lemma we have

$$
\begin{aligned}
2\|a\|_{L^{p^{\prime}(\cdot)(\Omega)}}+b+1 & \\
= & \liminf _{n \rightarrow \infty} \frac{\left(2\|a\|_{L^{p^{\prime}(\cdot)}(\Omega)}+b+1\right)\left(\int_{\Omega} F\left(x, u_{n}(x)\right) \mathrm{d} x+\int_{\partial \Omega} G\left(x, u_{n}(x)\right) \mathrm{d} S\right)}{\int_{\Omega} F\left(x, u_{n(x)}\right) \mathrm{d} x+\int_{\partial \Omega} G\left(x, u_{n}(x)\right) \mathrm{d} S+c-o(1)} \\
\geqslant & \liminf _{n \rightarrow \infty} \int_{\Omega} \frac{F\left(x, u_{n}(x)\right)}{\left\|u_{n}\right\|_{X}^{p_{+}}} \mathrm{d} x+\liminf _{n \rightarrow \infty} \int_{\partial \Omega} \frac{G\left(x, u_{n}(x)\right)}{\left\|u_{n}\right\|_{X}^{p_{+}}} \mathrm{d} S \\
= & \liminf _{n \rightarrow \infty} \int_{\Omega} \frac{F\left(x, u_{n}(x)\right)}{\left\|u_{n}\right\|_{X}^{p_{+}}} \mathrm{d} x-\limsup _{n \rightarrow \infty} \int_{\Omega} \frac{C_{3}}{\left\|u_{n}\right\|_{X}^{p_{+}}} \mathrm{d} x \\
& +\liminf _{n \rightarrow \infty} \int_{\partial \Omega} \frac{G\left(x, u_{n}(x)\right)}{\left\|u_{n}\right\|_{X}^{p_{+}}} \mathrm{d} S-\limsup _{n \rightarrow \infty} \int_{\partial \Omega} \frac{C_{4}}{\left\|u_{n}\right\|_{X}^{p_{+}}} \mathrm{d} S \\
= & \liminf _{n \rightarrow \infty} \int_{\Omega} \frac{F\left(x, u_{n}(x)\right)-C_{3}}{\left\|u_{n}\right\|_{X}^{p_{+}}} \mathrm{d} x+\liminf _{n \rightarrow \infty} \int_{\partial \Omega} \frac{G\left(x, u_{n}(x)\right)-C_{4}}{\left\|u_{n}\right\|_{X}^{p_{+}}} \mathrm{d} S \\
\geqslant & \liminf _{n \rightarrow \infty} \int_{\Omega \cap \Omega_{0}} \frac{F\left(x, u_{n}(x)\right)-C_{3}}{\left\|u_{n}\right\|_{X}^{p_{+}}} \mathrm{d} x+\liminf _{n \rightarrow \infty} \int_{\partial \Omega \cap \Omega_{0}} \frac{G\left(x, u_{n}(x)\right)-C_{4}}{\left\|u_{n}\right\|_{X}^{p_{+}}} \mathrm{d} S
\end{aligned}
$$




$$
\begin{aligned}
& \geqslant \int_{\Omega \cap \Omega_{0}} \liminf _{n \rightarrow \infty} \frac{F\left(x, u_{n}(x)\right)-C_{3}}{\left\|u_{n}\right\|_{X}^{p_{+}}} \mathrm{d} x+\int_{\partial \Omega \cap \Omega_{0}} \liminf _{n \rightarrow \infty} \frac{G\left(x, u_{n}(x)\right)-C_{4}}{\left\|u_{n}\right\|_{X}^{p_{+}}} \mathrm{d} S \\
& =\int_{\Omega \cap \Omega_{0}} \liminf _{n \rightarrow \infty} \frac{F\left(x, u_{n}(x)\right)}{\left|u_{n}(x)\right|^{p_{+}}}\left|w_{n}(x)\right|^{p_{+}} \mathrm{d} x-\int_{\Omega \cap \Omega_{0}} \limsup _{n \rightarrow \infty} \frac{C_{3}}{\left\|u_{n}\right\|_{X}^{p_{+}}} \mathrm{d} x \\
& \quad+\int_{\partial \Omega \cap \Omega_{0}} \liminf _{n \rightarrow \infty} \frac{G\left(x, u_{n}(x)\right)}{\left|u_{n}(x)\right|^{p_{+}}}\left|w_{n}(x)\right|^{p_{+}} \mathrm{d} S-\int_{\partial \Omega \cap \Omega_{0}} \limsup _{n \rightarrow \infty} \frac{C_{4}}{\left\|u_{n}\right\|_{X}^{p_{+}}} \mathrm{d} S \\
& =+\infty,
\end{aligned}
$$

which is a contradiction. Therefore, $\left|\Omega_{0}\right|=0$, and we have $w(x)=0$ almost everywhere in $\bar{\Omega}$.

Since $I\left(t u_{n}\right)$ is continuous for $t \in[0,1]$, for each $n \in \mathbb{N}$ there exists $t_{n} \in[0,1]$ such that

$$
I\left(t_{n} u_{n}\right):=\max _{t \in[0,1]} I\left(t u_{n}\right)
$$

Let $\left\{\varrho_{k}\right\}$ be a sequence of real numbers such that $\varrho_{k}>1$ for any $k$ and $\lim _{k \rightarrow \infty} \varrho_{k}=$ $+\infty$. Then $\left\|\varrho_{k} w_{n}\right\|_{X}=\varrho_{k}>1$ for all $k$ and $n$. For fixed $k$, since $w_{n} \rightarrow 0$ strongly in the spaces $L^{\alpha(\cdot)}(\Omega)$ and $L^{\beta(\cdot)}(\partial \Omega)$ as $n \rightarrow \infty$, it follows from the continuity of the Nemytskii operator that $F\left(x, \varrho_{k} w_{n}\right) \rightarrow 0$ in $L^{1}(\Omega)$ as $n \rightarrow \infty$, and $G\left(x, \varrho_{k} w_{n}\right) \rightarrow 0$ in $L^{1}(\partial \Omega)$ as $n \rightarrow \infty$; see [17, theorem 1.1]. We derive that

$$
\lim _{n \rightarrow \infty} \int_{\Omega} F\left(x, \varrho_{k} w_{n}\right) \mathrm{d} x=0
$$

and

$$
\lim _{n \rightarrow \infty} \int_{\partial \Omega} G\left(x, \varrho_{k} w_{n}\right) \mathrm{d} S=0 .
$$

Since $\left\|u_{n}\right\|_{X} \rightarrow \infty$ as $n \rightarrow \infty$, we obtain that $\left\|u_{n}\right\|_{X}>\varrho_{k}$ and so $0<\varrho_{k} /\left\|u_{n}\right\|_{X}<1$ for $n$ large enough. It follows from $(\mathrm{J} 4),(3.15),(3.16)$ and lemma 2.3 that

$$
\begin{aligned}
I\left(t_{n} u_{n}\right) \geqslant & I\left(\frac{\varrho_{k}}{\left\|u_{n}\right\|_{X}} u_{n}\right) \\
= & I\left(\varrho_{k} w_{n}\right) \\
= & \int_{\Omega} \Phi_{0}\left(x, \nabla \varrho_{k} w_{n}\right) \mathrm{d} x+\int_{\Omega} \frac{1}{p(x)}\left|\varrho_{k} w_{n}\right|^{p(x)} \mathrm{d} x-\int_{\Omega} F\left(x, \varrho_{k} w_{n}\right) \mathrm{d} x \\
& \quad-\int_{\partial \Omega} G\left(x, \varrho_{k} w_{n}\right) \mathrm{d} S \\
\geqslant & \frac{d}{p_{+}} \int_{\Omega}\left|\nabla \varrho_{k} w_{n}\right|^{p(x)} \mathrm{d} x+\frac{1}{p_{+}} \int_{\Omega}\left|\varrho_{k} w_{n}\right|^{p(x)} \mathrm{d} x-\int_{\Omega} F\left(x, \varrho_{k} w_{n}\right) \mathrm{d} x \\
& \quad-\int_{\partial \Omega} G\left(x, \varrho_{k} w_{n}\right) \mathrm{d} S \\
\geqslant & C_{5}\left\|\varrho_{k} w_{n}\right\|_{X}^{p_{-}}-\int_{\Omega} F\left(x, \varrho_{k} w_{n}\right) \mathrm{d} x-\int_{\partial \Omega} G\left(x, \varrho_{k} w_{n}\right) \mathrm{d} S \\
\geqslant & \frac{C_{5}}{2} \varrho_{k}^{p_{-}}
\end{aligned}
$$


for some positive constant $C_{5}$ and for any $n$ large enough. Letting $n, k \rightarrow \infty$ in relation (3.17) implies that

$$
\lim _{n \rightarrow \infty} I\left(t_{n} u_{n}\right)=+\infty
$$

For any $n$ large enough, it is obvious that $I\left(t_{n} u_{n}\right)>0=I(0)=I\left(0 u_{n}\right)$, and thus $t_{n}>0$. If $t_{n}<1$, then $\left.(\mathrm{d} / \mathrm{d} t) I\left(t u_{n}\right)\right|_{t=t_{n}}=0$, which implies that $\left\langle I^{\prime}\left(t_{n} u_{n}\right), t_{n} u_{n}\right\rangle=$ 0 . If $t_{n}=1$, then $\left\langle I^{\prime}\left(u_{n}\right), u_{n}\right\rangle=o(1)$. Hence, we always know that

$$
\left\langle I^{\prime}\left(t_{n} u_{n}\right), t_{n} u_{n}\right\rangle=o(1)
$$

for sufficiently large $n$. On the other hand, for all $n$ large enough, we deduce from assumptions (J5), (F4), (G3), (3.5) and (3.19) that

$$
\begin{aligned}
& I\left(t_{n} u_{n}\right)=I\left(t_{n} u_{n}\right)-\frac{1}{p_{+}}\left\langle I^{\prime}\left(t_{n} u_{n}\right), t_{n} u_{n}\right\rangle+o(1) \\
& =\int_{\Omega} \Phi_{0}\left(x, t_{n} \nabla u_{n}\right) \mathrm{d} x+\int_{\Omega} \frac{1}{p(x)}\left|t_{n} u_{n}\right|^{p(x)} \mathrm{d} x-\int_{\Omega} F\left(x, t_{n} u_{n}\right) \mathrm{d} x \\
& -\int_{\partial \Omega} G\left(x, t_{n} u_{n}\right) \mathrm{d} S-\frac{1}{p_{+}} \int_{\Omega} \varphi\left(x, t_{n} \nabla u_{n}\right) \cdot\left(t_{n} \nabla u_{n}\right) \mathrm{d} x \\
& -\frac{1}{p_{+}} \int_{\Omega}\left|t_{n} u_{n}\right|^{p(x)} \mathrm{d} x+\frac{1}{p_{+}} \int_{\Omega} f\left(x, t_{n} u_{n}\right) t_{n} u_{n} \mathrm{~d} x \\
& +\frac{1}{p_{+}} \int_{\partial \Omega} g\left(x, t_{n} u_{n}\right) t_{n} u_{n} \mathrm{~d} S+o(1) \\
& =\frac{1}{p_{+}} \int_{\Omega} \mathcal{H}\left(x, t_{n} \nabla u_{n}\right) \mathrm{d} x+\int_{\Omega} \frac{1}{p(x)}\left|t_{n} u_{n}\right|^{p(x)} \mathrm{d} x-\frac{1}{p_{+}} \int_{\Omega}\left|t_{n} u_{n}\right|^{p(x)} \mathrm{d} x \\
& +\frac{1}{p_{+}} \int_{\Omega} \mathcal{F}\left(x, t_{n} u_{n}\right) \mathrm{d} x+\frac{1}{p_{+}} \int_{\partial \Omega} \mathcal{G}\left(x, t_{n} u_{n}\right) \mathrm{d} S+o(1) \\
& \leqslant \frac{1}{p_{+}} \int_{\Omega}\left(\mathcal{H}\left(x, \nabla u_{n}\right)+\mu_{1}\right) \mathrm{d} x+\int_{\Omega} \frac{1}{p(x)}\left|u_{n}\right|^{p(x)} \mathrm{d} x-\frac{1}{p_{+}} \int_{\Omega}\left|u_{n}\right|^{p(x)} \mathrm{d} x \\
& +\frac{1}{p_{+}} \int_{\Omega}\left(\mathcal{F}\left(x, u_{n}\right)+\mu_{2}\right) \mathrm{d} x+\frac{1}{p_{+}} \int_{\partial \Omega}\left(\mathcal{G}\left(x, u_{n}\right)+\mu_{3}\right) \mathrm{d} S+o(1) \\
& \leqslant \int_{\Omega} \Phi_{0}\left(x, \nabla u_{n}\right) \mathrm{d} x+\int_{\Omega} \frac{1}{p(x)}\left|u_{n}\right|^{p(x)} \mathrm{d} x-\int_{\Omega} F\left(x, u_{n}\right) \mathrm{d} x \\
& -\int_{\partial \Omega} G\left(x, u_{n}\right) \mathrm{d} S-\frac{1}{p_{+}}\left(\int_{\Omega} \varphi\left(x, \nabla u_{n}\right) \cdot \nabla u_{n} \mathrm{~d} x+\int_{\Omega}\left|u_{n}\right|^{p(x)} \mathrm{d} x\right. \\
& \left.-\int_{\Omega} f\left(x, u_{n}\right) u_{n} \mathrm{~d} x-\int_{\partial \Omega} g\left(x, u_{n}\right) u_{n} \mathrm{~d} S\right) \\
& +o(1)+C_{6} \\
& =I\left(u_{n}\right)-\frac{1}{p_{+}}\left\langle I^{\prime}\left(u_{n}\right), u_{n}\right\rangle+o(1)+C_{6} \\
& \rightarrow c+C_{6} \quad \text { as } n \rightarrow \infty
\end{aligned}
$$

for some positive constant $C_{6}$. Due to (3.18), we have a contradiction, and thus the sequence $\left\{u_{n}\right\}$ is bounded in $X$. Therefore, the functional $I$ satisfies the $(\mathrm{C})_{c}$-condition for any $c \in \mathbb{R}$. 
It is well known that since $X$ is a reflexive and separable Banach space, there are $\left\{e_{n}\right\} \subseteq X$ and $\left\{f_{n}^{*}\right\} \subseteq X^{*}$ such that

$$
X=\overline{\operatorname{span}\left\{e_{n}: n=1,2, \ldots\right\}}, \quad X^{*}=\overline{\operatorname{span}\left\{f_{n}^{*}: n=1,2, \ldots\right\}},
$$

and

$$
\left\langle f_{i}^{*}, e_{j}\right\rangle= \begin{cases}1 & \text { if } i=j \\ 0 & \text { if } i \neq j\end{cases}
$$

Let us define $X_{k}=\operatorname{span}\left\{e_{k}\right\}, Y_{k}=\bigoplus_{m=1}^{k} X_{m}$ and $Z_{k}=\overline{\bigoplus_{m=k}^{\infty} X_{m}}$ for $k \in \mathbb{N}$. In order to establish the existence and multiplicity results, we use the following Fountain theorem.

Lemma 3.9 (see Willem [37]). Let $X$ be a real reflexive Banach space and let $I \in$ $C^{1}(X, \mathbb{R})$ satisfy the $(\mathrm{C})_{c^{-}}$-condition for any $c>0$ and I even. If for each sufficiently large $k \in \mathbb{N}$ there exist $\rho_{k}>\delta_{k}>0$ such that

(1) $b_{k}:=\inf \left\{I(u): u \in Z_{k},\|u\|_{X}=\delta_{k}\right\} \rightarrow \infty$ as $k \rightarrow \infty$,

(2) $a_{k}:=\max \left\{I(u): u \in Y_{k},\|u\|_{X}=\rho_{k}\right\} \leqslant 0$.

hold, then the functional I has an unbounded sequence of critical values, i.e. there exists a sequence $\left\{u_{n}\right\} \subset X$ such that $I^{\prime}\left(u_{n}\right)=0$ and $I\left(u_{n}\right) \rightarrow+\infty$ as $n \rightarrow+\infty$.

The following result is useful to prove our main theorem.

Lemma 3.10 (Fan [12]). Define

$$
\theta_{k}=\sup _{\|u\|_{X}=1, u \in Z_{k}}\|u\|_{L^{\alpha(\cdot)}(\Omega)} \quad \text { and } \quad \eta_{k}=\sup _{\|u\|_{X}=1, u \in Z_{k}}\|u\|_{L^{\beta(\cdot)}(\partial \Omega)},
$$

where $\alpha(x)$ and $\beta(x)$ were given in (F2) and (G1), respectively. Then $\lim _{k \rightarrow \infty} \theta_{k}=0$ and $\lim _{k \rightarrow \infty} \eta_{k}=0$.

Theorem 3.11. Assume that (J1)-(J5), (F1)-(F5) and (G1)-(G4) hold. Then if $\Phi_{0}(x,-v)=\Phi_{0}(x, v)$ holds for all $(x, v) \in \Omega \times \mathbb{R}^{N}$, then the energy functional $I$ has a sequence of critical points $\left\{ \pm u_{n}\right\}$ in $X$ such that $I_{\lambda}\left( \pm u_{n}\right) \rightarrow \infty$ as $n \rightarrow \infty$.

Proof. Obviously, $I$ is an even functional and satisfies the $(\mathrm{C})_{c}$-condition for any $c>0$. It is enough to show that there exist $\rho_{k}>\delta_{k}>0$ such that

(1) $b_{k}:=\inf \left\{I(u): u \in Z_{k},\|u\|_{X}=\delta_{k}\right\} \rightarrow \infty$ as $k \rightarrow \infty$,

(2) $a_{k}:=\max \left\{I(u): u \in Y_{k},\|u\|_{X}=\rho_{k}\right\} \leqslant 0$

for $k$ large enough. 
First of all, we prove condition (1). Assume that $\|u\|_{X}>1$. It follows from (J4), (F2), (G1) and lemmas 2.2 and 2.3 that

$$
\begin{aligned}
I(u)= & \int_{\Omega} \Phi_{0}(x, \nabla u) \mathrm{d} x+\int_{\Omega} \frac{1}{p(x)}|u|^{p(x)} \mathrm{d} x-\int_{\Omega} F(x, u) \mathrm{d} x-\int_{\partial \Omega} G(x, u) \mathrm{d} S \\
\geqslant & \frac{d}{p_{+}} \int_{\Omega}|\nabla u|^{p(x)} \mathrm{d} x+\frac{1}{p_{+}} \int_{\Omega}|u|^{p(x)} \mathrm{d} x-\int_{\Omega}\left(d_{1}|u|+\frac{d_{2}}{\alpha(x)}|u|^{\alpha(x)}\right) \mathrm{d} x \\
& -\int_{\partial \Omega}\left(d_{3}|u|+\frac{d_{4}}{\beta(x)}|u|^{\beta(x)}\right) \mathrm{d} S \\
\geqslant & \frac{\min \{d, 1\}}{p_{+}}\|u\|_{X}^{p_{-}}-\max \left\{d_{1}, d_{3}\right\}\|u\|_{X}-\frac{d_{2}}{\alpha_{-}} \max \left\{\|u\|_{L^{\alpha(\cdot)(\Omega)}}^{\alpha_{-}},\|u\|_{L^{\alpha(\cdot)(\Omega)}}^{\alpha_{+}}\right\} \\
& -\frac{d_{4}}{\beta_{-}} \max \left\{\|u\|_{L^{\beta(\cdot)}(\partial \Omega)}^{\beta_{-}},\|u\|_{L^{\beta(\cdot)}(\partial \Omega)}^{\beta_{+}}\right\} \\
\geqslant & \frac{\min \{d, 1\}}{p_{+}}\|u\|_{X}^{p_{-}}-\max \left\{d_{1}, d_{3}\right\}\|u\|_{X} \\
& -C_{7} \max \left\{\|u\|_{L^{\alpha(\cdot)}(\Omega)}^{\alpha_{+}},\|u\|_{L^{\alpha(\cdot)}(\Omega)}^{\alpha_{-}},\|u\|_{L^{\beta(\cdot)}(\partial \Omega)}^{\beta_{+}},\|u\|_{L^{\beta(\cdot)}(\partial \Omega)}^{\beta_{-}}\right\}
\end{aligned}
$$

for some positive constant $C_{7}$. Put

$$
\|u\|_{L^{\alpha(\cdot)}(\Omega)}^{\alpha_{+}}=\max \left\{\|u\|_{L^{\alpha(\cdot)}(\Omega)}^{\alpha_{+}},\|u\|_{L^{\alpha(\cdot)}(\Omega)}^{\alpha_{-}},\|u\|_{L^{\beta(\cdot)}(\partial \Omega)}^{\beta_{+}},\|u\|_{L^{\beta(\cdot)}(\partial \Omega)}^{\beta_{-}}\right\} .
$$

Then we have

$$
I(u) \geqslant \frac{\min \{d, 1\}}{p_{+}}\|u\|_{X}^{p_{-}}-\max \left\{d_{1}, d_{3}\right\}\|u\|_{X}-C_{7} \theta_{k}^{\alpha_{+}}\|u\|_{X}^{\alpha_{+}} .
$$

Choose $\delta_{k}=\left(\alpha_{+} C_{7} \theta_{k}^{\alpha_{+}} / \min \{d, 1\}\right)^{1 /\left(p_{-}-\alpha_{+}\right)}$. It is clear that $\delta_{k} \rightarrow \infty$ as $k \rightarrow \infty$ because $p_{-}<\alpha_{+}$and $\theta_{k} \rightarrow 0$ as $k \rightarrow \infty$ by lemma 3.10. Therefore, if $u \in Z_{k}$ and $\|u\|_{X}=\delta_{k}$, we have

$$
I(u) \geqslant \frac{\min \{d, 1\}}{2}\left(\frac{1}{p_{+}}-\frac{1}{\alpha_{+}}\right) \delta_{k}^{p_{-}}-\max \left\{d_{1}, d_{3}\right\} \delta_{k} \rightarrow \infty \quad \text { as } k \rightarrow \infty .
$$

Like the previous argument, $I(u) \rightarrow \infty$ as $k \rightarrow \infty$ in the other three cases since $p_{-}<\alpha_{-} \leqslant \alpha_{+}, p_{-}<\beta_{-} \leqslant \beta_{+}$and $\theta_{k}, \eta_{k} \rightarrow 0$ as $k \rightarrow \infty$ by lemma 3.10.

Next we prove condition (2). Let $u \in Y_{k}$ and $\|u\|_{X}>1$. By assumptions (F3) and (G2), we see that for any $M>0$ there exist two positive constants, both denoted by $C(M)$, which depend on $M$ and satisfy

$$
F(x, t) \geqslant M|t|^{p_{+}}-C(M) \text { for all }(x, t) \in \Omega \times \mathbb{R}
$$

and

$$
G(x, t) \geqslant M|t|^{p_{+}}-C(M) \text { for all }(x, t) \in \partial \Omega \times \mathbb{R} .
$$


For $k>1$, it follows from (J2), (3.20), (3.21) and lemmas 2.1 and 2.3 that

$$
\begin{aligned}
I(u)= & \int_{\Omega} \Phi_{0}(x, \nabla u) \mathrm{d} x+\int_{\Omega} \frac{1}{p(x)}|u|^{p(x)} \mathrm{d} x-\int_{\Omega} F(x, u) \mathrm{d} x-\int_{\partial \Omega} G(x, u) \mathrm{d} S \\
\leqslant & \int_{\Omega} a(x)|\nabla u| \mathrm{d} x+\int_{\Omega} \frac{1}{p(x)} b|\nabla u|^{p(x)} \mathrm{d} x+\int_{\Omega} \frac{1}{p(x)}|u|^{p(x)} \mathrm{d} x \\
& \quad-\int_{\Omega}\left\{M|u|^{p_{+}}-C(M)\right\} \mathrm{d} x-\int_{\partial \Omega}\left\{M|u|^{p_{+}}-C(M)\right\} \mathrm{d} S \\
\leqslant & 2\|a\|_{L^{p^{\prime}(\cdot)(\Omega)}}\|\nabla u\|_{L^{p(\cdot)}(\Omega)}+(b+1)\|u\|_{X}^{p_{+}}-M \int_{\Omega}|u|^{p_{+}} \mathrm{d} x \\
& \quad-M \int_{\partial \Omega}|u|^{p_{+}} \mathrm{d} S+C(M)(|\Omega|+|\partial \Omega|) \\
\leqslant & \left(2\|a\|_{L^{p^{\prime}(\cdot)(\Omega)}}+b+1\right)\|u\|_{X}^{p_{+}}-M\left(\int_{\Omega}|u|^{p_{+}} \mathrm{d} x+\int_{\partial \Omega}|u|^{p_{+}} \mathrm{d} S\right)+C_{8}
\end{aligned}
$$

for some positive constant $C_{8}$. Since $\operatorname{dim} Y_{k}<\infty$, all norms are equivalent in $Y_{k}$. So

$$
I(u) \leqslant\left(2\|a\|_{L^{p^{\prime}(\cdot)}(\Omega)}+b+1\right)\|u\|_{X}^{p_{+}}-M C_{9}\|u\|_{X}^{p_{+}}+C_{8}
$$

for some positive constant $C_{9}$. If $M$ is large enough such that $\left(2\|a\|_{L^{p^{\prime}(\cdot)(\Omega)}}+b+\right.$ $1)<M C_{9}$, then we obtain $I(u) \rightarrow-\infty$ as $\|u\|_{X} \rightarrow \infty$, and thus we can choose $\rho_{k}>\delta_{k}>0$.

\section{Existence of infinitely many weak solutions converging to zero}

In this section we prove the existence of infinitely many weak solutions for the nonlinear Neumann boundary-value problem (P) using the argument in $[34,36]$ (theorem 4.7 below). For this, we employ the regularity lemma (lemma 4.3) and the cut-off method (lemma 4.6). First of all, we need the following additional assumptions on $\varphi, \Phi_{0}, f$ and $g$.

(J6) $\varphi(x, v) \cdot v-p_{-} \Phi_{0}(x, v) \geqslant 0$ for all $x \in \bar{\Omega}$ and for all $v \in \mathbb{R}^{N}$.

(F6) There exists a constant $s_{0}>0$ such that $p_{-} F(x, t)-f(x, t) t>0$ for all $x \in \bar{\Omega}$ and for $0<|t| \leqslant s_{0}$.

(F7) $\lim _{|t| \rightarrow 0}\left(f(x, t) /|t|^{p_{-}-2} t\right)=+\infty$ uniformly for all $x \in \Omega$.

(G5) There exists a constant $s_{1}>0$ such that $p_{-} G(x, t)-g(x, t) t>0$ for all $x \in \partial \Omega$ and for $0<|t| \leqslant s_{1}$.

(G6) $\lim _{|t| \rightarrow 0}\left(g(x, t) /|t|^{p_{-}-2} t\right)=+\infty$ uniformly for all $x \in \partial \Omega$.

Let us introduce the following lemma, which will be useful in the proof of a result about regularity for a weak solution of problem $(\mathrm{P})$. Define $u_{+}=\max \{u, 0\}$ and $u_{-}=-\min \{u, 0\}$.

Remark 4.1. If $u \in X$ and $k \geqslant 0$, then $(u-k)_{+},(u+k)_{-} \in X$.

To apply De Giorgi's technique, we need the following crucial lemma. The proof is given in [21, lemma 4.3], which is an extension of [35, lemma 2.2]. 
LEMMA 4.2. Let $\left\{Z_{n}\right\}_{n=1}^{\infty}$ be a sequence of positive numbers satisfying the recursion inequality

$$
Z_{n+1} \leqslant k b^{n}\left(Z_{n}^{1+\delta_{1}}+Z_{n}^{1+\delta_{2}}\right), \quad n=0,1,2, \ldots,
$$

for some $b>1, k>0$ and $\delta_{2} \geqslant \delta_{1}>0$. If $Z_{0} \leqslant \min \left\{1,(2 k)^{(-1) / \delta_{1}} b^{(-1) / \delta_{1}^{2}}\right\}$ or

$$
Z_{0} \leqslant \min \left\{(2 k)^{(-1) / \delta_{1}} b^{(-1) / \delta_{1}^{2}},(2 k)^{(-1) / \delta_{2}} b^{-1 /\left(\delta_{1} \delta_{2}\right)-\left(\delta_{2}-\delta_{1}\right) / \delta_{2}^{2}}\right\},
$$

then $Z_{n} \leqslant 1$ for some $n \in \mathbb{N} \cup\{0\}$. Moreover,

$$
Z_{n} \leqslant \min \left\{1,(2 k)^{(-1) / \delta_{1}} b^{(-1) / \delta_{1}^{2}} b^{(-n) / \delta_{1}}\right\}
$$

for any $n \geqslant n_{0}$, where $n_{0}$ is the smallest $n \in \mathbb{N} \cup\{0\}$ satisfying $Z_{n} \leqslant 1$. In particular, $Z_{n} \rightarrow 0$ as $n \rightarrow \infty$.

Next, from an analogous argument to that in [20, theorem 4.2], we prove proposition 4.3, which is a regularity-type lemma, via De Giorgi's technique and the localization method. We point out that proposition 4.3 is reproved in the present circumstances because the lemma 4.2 above is slightly different from [39, lemma 2.1], even though their method was applied to the Neumann problem with a nonlinear boundary condition.

Proposition 4.3. Assume that (J1), (J2), (J4), (F1), (F2) and (G1) hold. Then there exist positive constants $\eta, \rho$ such that if $u$ is a weak solution of problem $(P)$, then

$$
\begin{aligned}
-\eta\left[1+\left(\int_{\Omega}(-u)_{+}^{\alpha(x)} \mathrm{d} x+\int_{\partial \Omega}\right.\right. & \left.\left.(-u)_{+}^{\beta(x)} \mathrm{d} S\right)^{\rho}\right] \\
& \leqslant \operatorname{essinf}_{\bar{\Omega}} u \\
& \leqslant \operatorname{ess} \sup _{\bar{\Omega}} \\
& \leqslant \eta\left[1+\left(\int_{\Omega} u_{+}^{\alpha(x)} \mathrm{d} x+\int_{\partial \Omega} u_{+}^{\beta(x)} \mathrm{d} S\right)^{\rho}\right],
\end{aligned}
$$

that is, $u \in L^{\infty}(\bar{\Omega})$.

Proof. Let $A_{k}=\{x \in \bar{\Omega}: u(x)>k\}, \tilde{A_{k}}=\{x \in \bar{\Omega}:-u(x)>k\}, k \in \mathbb{N}$. Taking $v=(u-k)_{+} \in X$ as a test function in $(\mathrm{P})$ and integrating over $\Omega$, we have

$$
\int_{\Omega} \varphi(x, \nabla u) \cdot \nabla v \mathrm{~d} x+\int_{\Omega}|u|^{p(x)-2} u v \mathrm{~d} x=\int_{\Omega} f(x, u) v \mathrm{~d} x+\int_{\partial \Omega} g(x, u) v \mathrm{~d} S .
$$

Equivalently,

$$
\begin{aligned}
\int_{\Omega \cap A_{k}} \varphi(x, \nabla u) \cdot \nabla u \mathrm{~d} x & +\int_{\Omega \cap A_{k}}|u|^{p(x)-2} u(u-k) \mathrm{d} x \\
= & \int_{\Omega \cap A_{k}} f(x, u)(u-k) \mathrm{d} x+\int_{\partial \Omega \cap A_{k}} g(x, u)(u-k) \mathrm{d} S .
\end{aligned}
$$


Hence, since $u \geqslant u-k>0$ and $u \geqslant k \geqslant 1$ on $A_{k}$, by (F2) and (G1),

$$
\begin{aligned}
\int_{\Omega \cap A_{k}} \varphi(x, \nabla u) & \cdot \nabla u \mathrm{~d} x \\
\leqslant & -\int_{\Omega \cap A_{k}}|u|^{p(x)-2} u(u-k) \mathrm{d} x+\int_{\Omega \cap A_{k}}\left(d_{1}+d_{2}|u|^{\alpha(x)-1}\right)(u-k) \mathrm{d} x \\
& \quad+\int_{\partial \Omega \cap A_{k}}\left(d_{3}+d_{4}|u|^{\beta(x)-1}\right)(u-k) \mathrm{d} S \\
\leqslant & \int_{\Omega \cap A_{k}}\left(d_{1}+d_{2}|u|^{\alpha(x)-1}\right) u \mathrm{~d} x+\int_{\partial \Omega \cap A_{k}}\left(d_{3}+d_{4}|u|^{\beta(x)-1}\right) u \mathrm{~d} S \\
\leqslant & \left(d_{1}+d_{2}\right) \int_{\Omega \cap A_{k}} u^{\alpha(x)} \mathrm{d} x+\left(d_{3}+d_{4}\right) \int_{\partial \Omega \cap A_{k}} u^{\beta(x)} \mathrm{d} S .
\end{aligned}
$$

Similarly, taking $v=-(u+k)_{-}=-(-u-k)_{+} \in X$ as a test function in $(\mathrm{P})$ we obtain

$\int_{\Omega \cap \tilde{A}_{k}} \varphi(x, \nabla u) \cdot \nabla u \mathrm{~d} x \leqslant\left(d_{1}+d_{2}\right) \int_{\Omega \cap \tilde{A}_{k}}(-u)^{\alpha(x)} \mathrm{d} x+\left(d_{3}+d_{4}\right) \int_{\partial \Omega \cap \tilde{A}_{k}}(-u)^{\beta(x)} \mathrm{d} S$.

Put $k_{n}:=k_{*}\left(2-1 / 2^{n}\right), n=0,1,2, \ldots$, with $k_{*} \geqslant 1$ specified later, and

$$
Z_{n}:=\int_{\Omega \cap A_{k_{n}}}\left(u-k_{n}\right)^{\alpha(x)} \mathrm{d} x+\int_{\partial \Omega \cap A_{k_{n}}}\left(u-k_{n}\right)^{\beta(x)} \mathrm{d} S .
$$

Note that $k_{*} \leqslant k_{n} \leqslant k_{n+1} \leqslant 2 k_{*}$ for all $n \in \mathbb{N}$. Recalling the definition of $k_{n}$, we have

$$
\begin{aligned}
\int_{\Omega \cap A_{k_{n}}}\left(u-k_{n}\right)^{\alpha(x)} \mathrm{d} x & \geqslant \int_{\Omega \cap A_{k_{n+1}}} u^{\alpha(x)}\left(1-\frac{k_{n}}{k_{n+1}}\right)^{\alpha(x)} \mathrm{d} x \\
& \geqslant \int_{\Omega \cap A_{k_{n+1}}} \frac{u^{\alpha(x)}}{2^{\alpha(x)(n+2)}} \mathrm{d} x
\end{aligned}
$$

and

$$
\begin{aligned}
Z_{n} & \geqslant \int_{\Omega \cap A_{k_{n+1}}} \frac{u^{\alpha(x)}}{2^{\alpha(x)(n+2)}} \mathrm{d} x+\int_{\partial \Omega \cap A_{k_{n+1}}} \frac{u^{\beta(x)}}{2^{\beta(x)(n+2)}} \mathrm{d} S \\
& \geqslant \frac{1}{2^{\alpha+(n+2)}} \int_{\Omega \cap A_{k_{n+1}}} u^{\alpha(x)} \mathrm{d} x+\frac{1}{2^{\beta+(n+2)}} \int_{\partial \Omega \cap A_{k_{n+1}}} u^{\beta(x)} \mathrm{d} S \\
& \geqslant \frac{1}{2^{\max \left\{\alpha_{+}, \beta_{+}\right\}(n+2)}}\left(\int_{\Omega \cap A_{k_{n+1}}} u^{\alpha(x)} \mathrm{d} x+\int_{\partial \Omega \cap A_{k_{n+1}}} u^{\beta(x)} \mathrm{d} S\right) .
\end{aligned}
$$

Thus,

$$
\int_{\Omega \cap A_{k_{n+1}}} u^{\alpha(x)} \mathrm{d} x+\int_{\partial \Omega \cap A_{k_{n+1}}} u^{\beta(x)} \mathrm{d} S \leqslant e_{1}^{n+2} Z_{n}
$$


where $e_{1}=2^{\max \left\{\alpha_{+}, \beta_{+}\right\}}>1$. It follows from (J4), (4.1) and (4.3) that

$$
\begin{aligned}
\int_{\Omega \cap A_{k_{n+1}}} d \mid \nabla( & \left.u-k_{n+1}\right)\left.\right|^{p(x)} \mathrm{d} x \\
& \leqslant \int_{\Omega \cap A_{k_{n+1}}} \varphi\left(x, \nabla\left(u-k_{n+1}\right)\right) \cdot \nabla\left(u-k_{n+1}\right) \mathrm{d} x \\
& \leqslant\left(d_{1}+d_{2}\right) \int_{\Omega \cap A_{k_{n+1}}} u^{\alpha(x)} \mathrm{d} x+\left(d_{3}+d_{4}\right) \int_{\partial \Omega \cap A_{k_{n+1}}} u^{\beta(x)} \mathrm{d} S \\
& \leqslant\left(d_{1}+d_{2}+d_{3}+d_{4}\right) e_{1}^{n+2} Z_{n} .
\end{aligned}
$$

For the Lebesgue measure of $A_{k_{n+1}}$, we estimate

$$
\begin{aligned}
\left|A_{k_{n+1}}\right| \leqslant & \int_{\Omega \cap A_{k_{n+1}}}\left(\frac{u-k_{n}}{k_{n+1}-k_{n}}\right)^{\alpha(x)} \mathrm{d} x+\int_{\partial \Omega \cap A_{k_{n+1}}}\left(\frac{u-k_{n}}{k_{n+1}-k_{n}}\right)^{\beta(x)} \mathrm{d} S \\
\leqslant & \int_{\Omega \cap A_{k_{n+1}}}\left(\frac{2^{n+1}}{k_{*}}\right)^{\alpha(x)}\left(u-k_{n}\right)^{\alpha(x)} \mathrm{d} x \\
& +\int_{\partial \Omega \cap A_{k_{n+1}}}\left(\frac{2^{n+1}}{k_{*}}\right)^{\beta(x)}\left(u-k_{n}\right)^{\beta(x)} \mathrm{d} S \\
\leqslant & \frac{2^{(n+1) \alpha_{+}}}{k_{*}^{\alpha-}} \int_{\Omega \cap A_{k_{n+1}}}\left(u-k_{n}\right)^{\alpha(x)} \mathrm{d} x+\frac{2^{(n+1) \beta_{+}}}{k_{*}^{\beta-}} \int_{\partial \Omega \cap A_{k_{n+1}}}\left(u-k_{n}\right)^{\beta(x)} \mathrm{d} S \\
\leqslant & \left.\frac{2^{(n+1) \max \left\{\alpha_{+}, \beta_{+}\right\}}}{k_{*}}\left(u-k_{n}\right)^{\alpha(x)} \mathrm{d} x+\int_{\partial \Omega \cap A_{k_{n+1}}}\left(u-k_{n}\right)^{\beta(x)} \mathrm{d} S\right) .
\end{aligned}
$$

So

$$
\left|A_{k_{n+1}}\right| \leqslant \frac{e_{1}^{n+1}}{k_{*}} Z_{n} .
$$

The compactness of $\bar{\Omega}$ implies that for any $R>0$, there exists a finite open cover $\left\{B_{i}(R)\right\}_{i=1}^{m}$ of balls $B_{i}:=B_{i}(R)$ with radius $R$ such that $\bar{\Omega} \subset \bigcup_{i=1}^{m} B_{i}$. Define

$$
\begin{gathered}
p_{i}^{+}=\max _{\overline{B_{i}} \cap \bar{\Omega}} p(x), \quad \alpha_{i}^{+}=\max _{\overline{B_{i}} \cap \bar{\Omega}} \alpha(x), \quad \beta_{i}^{+}=\max _{\overline{B_{i}} \cap \bar{\Omega}} \beta(x), \\
p_{i}^{-}=\min _{\overline{B_{i}} \cap \bar{\Omega}} p(x), \quad \alpha_{i}^{-}=\min _{\overline{B_{i}} \cap \bar{\Omega}} \alpha(x), \quad \beta_{i}^{-}=\min _{\overline{B_{i}} \cap \bar{\Omega}} \beta(x), \\
\left(p_{i}^{-}\right)^{*}=\left\{\begin{array}{ll}
\frac{N p_{i}^{-}}{N-p_{i}^{-}} & \text {if } N>p_{i}^{-}, \\
+\infty & \text { if } N \leqslant p_{i}^{-},
\end{array} \quad\left(p_{i}^{-}\right)^{\partial}= \begin{cases}\frac{(N-1) p_{i}^{-}}{N-p_{i}^{-}} & \text {if } N>p_{i}^{-}, \\
+\infty & \text { if } N \leqslant p_{i}^{-} .\end{cases} \right.
\end{gathered}
$$

Since $p(x) \leqslant \alpha(x)<p^{*}(x)$ for all $x \in \bar{\Omega}, p(x) \leqslant \beta(x)<p^{\partial}(x)$ for all $x \in \partial \Omega$, and $p \in C(\bar{\Omega}), \alpha \in C_{+}(\bar{\Omega}), \beta \in C_{+}(\partial \Omega)$, we may take a sufficiently small $R>0$ such that for all $i \in\{1, \ldots, m\}$ we have

$$
p_{i}^{+} \leqslant \alpha_{i}^{+}<\left(p_{i}^{-}\right)^{*} \text { and } p_{i}^{+} \leqslant \beta_{i}^{+}<\left(p_{i}^{-}\right)^{\partial} .
$$


CASE $1\left(p_{i}^{+} \leqslant \alpha_{i}^{+}<\left(p_{i}^{-}\right)^{*}\right)$. In this case, $p_{i}^{+} \leqslant \alpha_{i}^{+}$is clear and if $p(x) \geqslant N$ for all $x \in \bar{\Omega}$, then $\alpha_{i}^{+}<\left(p_{i}^{-}\right)^{*}$ is obvious. For the case in which $p(x)<N$, we can modify the proof in [20] to get $\alpha_{i}^{+}<\left(p_{i}^{-}\right)^{*}$. For the convenience of the reader, we prove it. Assume that there exists $x \in \bar{\Omega}$ such that $p(x)<N$. Then we have

$$
\inf _{x \in \bar{\Omega}}\left(p^{*}(x)-\alpha(x)\right)=\delta_{1}>0
$$

for some $\delta_{1}>0$. Since $p(x)$ is uniformly continuous on $\bar{\Omega}$, for a sufficiently small $R>0$ we have

$$
|p(x)-p(y)| \leqslant \varepsilon_{1} \quad \text { for all } x, y \in \overline{B_{i}} \cap \bar{\Omega},
$$

where

$$
\varepsilon_{1}=\min \left\{\frac{N^{2} \alpha_{+}}{\left(N+\alpha_{+}\right)\left(N+2 \alpha_{+}\right)}, \frac{\delta_{1} N^{2}}{2\left(N+\alpha_{+}\right)\left(N+2 \alpha_{+}\right)}\right\} .
$$

Fix $i \in\{1, \ldots, m\}$. Defining $x_{i} \in \overline{B_{i}} \cap \bar{\Omega}$ such that $p\left(x_{i}\right)=p_{i}^{-}$, we consider the following three cases. The first, the $p\left(x_{i}\right) \geqslant N$ case, is obvious. For the second, that in which $N \alpha_{+} /\left(N+\alpha_{+}\right)<p\left(x_{i}\right)<N$, we have

$$
\left(p_{i}^{-}\right)^{*}=\frac{N p\left(x_{i}\right)}{N-p\left(x_{i}\right)}=-N+\frac{N^{2}}{N-p\left(x_{i}\right)}>-N+\frac{N^{2}}{N-N \alpha_{+} /\left(N+\alpha_{+}\right)}=\alpha_{+} \geqslant \alpha_{i}^{+} .
$$

For the final case, in which $p\left(x_{i}\right) \leqslant N \alpha_{+} /\left(N+\alpha_{+}\right)$, for all $y \in \overline{B_{i}} \cap \bar{\Omega}$ we have

$$
\begin{aligned}
p(y)<p\left(x_{i}\right)+\varepsilon_{1} \leqslant \frac{N \alpha_{+}}{N+\alpha_{+}}+\varepsilon_{1} & \leqslant \frac{N \alpha_{+}\left(N+2 \alpha_{+}\right)}{\left(N+\alpha_{+}\right)\left(N+2 \alpha_{+}\right)}+\frac{N^{2} \alpha_{+}}{\left(N+\alpha_{+}\right)\left(N+2 \alpha_{+}\right)} \\
& =\frac{2 N \alpha_{+}}{N+2 \alpha_{+}} \\
& <N
\end{aligned}
$$

Writing $\alpha_{i}^{+}=\alpha\left(y_{i}\right)$ for some $y_{i} \in \overline{B_{i}} \cap \bar{\Omega}$, we have

$$
\begin{aligned}
\left|p^{*}\left(y_{i}\right)-p^{*}\left(x_{i}\right)\right| & =\left|\frac{N^{2}}{N-p\left(y_{i}\right)}-N-\frac{N^{2}}{N-p\left(x_{i}\right)}+N\right| \\
& =N^{2} \frac{\left|p\left(y_{i}\right)-p\left(x_{i}\right)\right|}{\left(N-p\left(y_{i}\right)\right)\left(N-p\left(x_{i}\right)\right)} \\
& <N^{2} \frac{\varepsilon_{1}}{\left(N-2 N \alpha_{+}\left(N+2 \alpha_{+}\right)\right)\left(N-N \alpha^{+} /\left(N+\alpha_{+}\right)\right)} \\
& =\varepsilon_{1} N^{2} \frac{\left(N+2 \alpha_{+}\right)\left(N+\alpha_{+}\right)}{N^{4}} \\
& \leqslant \frac{\delta_{1}}{2} .
\end{aligned}
$$

Therefore, we get

$$
\alpha_{i}^{+}=\alpha\left(y_{i}\right) \leqslant p^{*}\left(y_{i}\right)-\delta_{1} \leqslant p^{*}\left(x_{i}\right)+\frac{\delta_{1}}{2}-\delta_{1}<p^{*}\left(x_{i}\right)=\left(p_{i}^{-}\right)^{*} .
$$


CASE $2\left(p_{i}^{+} \leqslant \beta_{i}^{+}<\left(p_{i}^{-}\right)^{\partial}\right)$. In this case, $p_{i}^{+} \leqslant \beta_{i}^{+}$is clear and if $p(x) \geqslant N$ for all $x \in \partial \Omega$, then $\beta_{i}^{+}<\left(p_{i}^{-}\right)^{\partial}$ is obvious. Assume that there exists $x \in \partial \Omega$ such that $p(x)<N$. Then we have

$$
\inf _{x \in \partial \Omega}\left(p^{\partial}(x)-\beta(x)\right)=\delta_{2}>0
$$

for some $\delta_{2}>0$. Since $p(x)$ is uniformly continuous on $\partial \Omega$, for a sufficiently small $R>0$ we have

$$
|p(x)-p(y)| \leqslant \varepsilon_{2} \quad \text { for all } x, y \in \overline{B_{i}} \cap \partial \Omega
$$

where

$$
\varepsilon_{2}=\min \left\{\frac{N \beta_{+}(N-2)}{\left(N-1+\beta_{+}\right)\left(N+2 \beta_{+}\right)}, \frac{\delta_{2} N^{2}}{2\left(N-1+\beta_{+}\right)\left(N+2 \beta_{+}\right)}\right\} .
$$

Fix $i \in\{1, \ldots, m\}$. Defining $x_{i} \in \overline{B_{i}} \cap \partial \Omega$ such that $p\left(x_{i}\right)=p_{i}^{-}$, we consider the following three cases. The first, the $p\left(x_{i}\right) \geqslant N$ case, is obvious. For the second, that in which $N \beta_{+} /\left(N-1+\beta_{+}\right)<p\left(x_{i}\right)<N$, we have $\left(N \beta_{+}+p\left(x_{i}\right)\right) /\left(\beta_{+}+N\right)<p\left(x_{i}\right)$ and

$$
\begin{aligned}
\left(p_{i}^{-}\right)^{\partial}=\frac{(N-1) p\left(x_{i}\right)}{N-p\left(x_{i}\right)} & =-N+\frac{N^{2}-p\left(x_{i}\right)}{N-p\left(x_{i}\right)} \\
& >-N+\frac{N^{2}-p\left(x_{i}\right)}{N-\left(N \beta_{+}+p\left(x_{i}\right)\right) /\left(\beta_{+}+N\right)} \\
& =\beta_{+} \\
& \geqslant \beta_{i}^{+} .
\end{aligned}
$$

For the final case, in which $p\left(x_{i}\right) \leqslant N \beta_{+} /\left(N-1+\beta_{+}\right)$, for all $y \in \overline{B_{i}} \cap \partial \Omega$ we have

$$
\begin{aligned}
p(y)<p\left(x_{i}\right)+\varepsilon_{2} & \leqslant \frac{N \beta_{+}}{N-1+\beta_{+}}+\varepsilon_{2} \\
& \leqslant \frac{N \beta_{+}\left(N+2 \beta_{+}\right)}{\left(N-1+\beta_{+}\right)\left(N+2 \beta_{+}\right)}+\frac{N \beta_{+}(N-2)}{\left(N-1+\beta_{+}\right)\left(N+2 \beta_{+}\right)} \\
& =\frac{2 N \beta_{+}}{N+2 \beta_{+}} \\
& <N .
\end{aligned}
$$

Denoting $\beta_{i}^{+}=\beta\left(y_{i}\right)$ for some $y_{i} \in \overline{B_{i}} \cap \partial \Omega$, we have

$$
\begin{aligned}
& \left|p^{\partial}\left(y_{i}\right)-p^{\partial}\left(x_{i}\right)\right|=\left|\frac{N^{2}-p\left(y_{i}\right)}{N-p\left(y_{i}\right)}-\frac{N^{2}-p\left(x_{i}\right)}{N-p\left(x_{i}\right)}\right| \\
& =\left|\frac{\begin{array}{c}
\left(N^{3}-p\left(x_{i}\right) N^{2}-p\left(y_{i}\right) N+p\left(y_{i}\right) p\left(x_{i}\right)\right) \\
-\left(N^{3}-p\left(y_{i}\right) N^{2}-p\left(x_{i}\right) N+p\left(x_{i}\right) p\left(y_{i}\right)\right)
\end{array}}{\left(N-p\left(y_{i}\right)\right)\left(N-p\left(x_{i}\right)\right)}\right| \\
& =\left|\frac{\left(N^{2}-N\right)\left(p\left(y_{i}\right)-p\left(x_{i}\right)\right)}{\left(N-p\left(y_{i}\right)\right)\left(N-p\left(x_{i}\right)\right)}\right| \\
& <N(N-1) \frac{\varepsilon_{2}}{\left(N-2 N \beta_{+} /\left(N+2 \beta_{+}\right)\right)\left(N-N \beta_{+} /\left(N-1+\beta_{+}\right)\right)}
\end{aligned}
$$




$$
\begin{aligned}
& =\varepsilon_{2} \frac{\left(N+2 \beta_{+}\right)\left(N-1+\beta_{+}\right)}{N^{2}} \\
& \leqslant \frac{\delta_{2}}{2} .
\end{aligned}
$$

Therefore, we get

$$
\beta_{i}^{+}=\beta\left(y_{i}\right) \leqslant p^{\partial}\left(y_{i}\right)-\delta_{2} \leqslant p^{\partial}\left(x_{i}\right)+\frac{\delta_{2}}{2}-\delta_{2}<p^{\partial}\left(x_{i}\right)=\left(p_{i}^{-}\right)^{\partial} .
$$

By way of cases 1 and 2, we show inequality (4.6). Next, to obtain the relation between $Z_{n}$ and $Z_{n+1}$, we choose a partition of unity $\left\{\xi_{i}\right\}_{i=1}^{m} \subset C_{0}^{\infty}\left(\mathbb{R}^{N}\right)$ associated with an open cover $\left\{B_{i}\right\}_{i=1}^{m}$, that is, we have

$$
\operatorname{supp} \xi_{i} \subset B_{i}, \quad 0 \leqslant \xi_{i} \leqslant 1, \quad \sum_{i=1}^{m} \xi_{i}=1 .
$$

Observe that

$$
\begin{aligned}
\int_{\Omega \cap A_{k_{n+1}}} d\left|\nabla\left(u-k_{n+1}\right)\right|^{p(x)} \mathrm{d} x & \\
& =\int_{\Omega \cap A_{k_{n+1}}} d\left|\nabla\left(u-k_{n+1}\right)\right|^{p(x)} \sum_{i=1}^{m} \xi_{i} \mathrm{~d} x \\
& \geqslant \sum_{i=1}^{m} \int_{\Omega \cap A_{k_{n+1}}} d\left\{\left|\nabla\left(u-k_{n+1}\right)\right|^{p_{i}^{-}}-1\right\} \xi_{i} \mathrm{~d} x \\
& \geqslant \sum_{i=1}^{m} \int_{\Omega \cap A_{k_{n+1}}} d\left|\nabla\left(u-k_{n+1}\right)\right|^{p_{i}^{-}} \xi_{i}^{p_{i}^{-}} \mathrm{d} x-d\left|\Omega \cap A_{k_{n+1}}\right|
\end{aligned}
$$

Therefore, it follows from the above inequality, (4.4) and (4.5) that we obtain

$$
\begin{aligned}
\sum_{i=1}^{m} \int_{\Omega \cap A_{k_{n+1}}} d\left|\xi_{i} \nabla\left(u-k_{n+1}\right)\right|^{p_{i}^{-}} \mathrm{d} x & \leqslant\left(d_{1}+d_{2}+d_{3}+d_{4}\right) e_{1}^{n+2} Z_{n}+d\left|\Omega \cap A_{k_{n+1}}\right| \\
& \leqslant\left(d_{1}+d_{2}+d_{3}+d_{4}\right) e_{1}^{n+2} Z_{n}+\frac{d e_{1}^{n+1}}{k_{*}} Z_{n} \\
& =e_{2} e_{1}^{n} Z_{n},
\end{aligned}
$$

where $e_{2}:=\left(d_{1}+d_{2}+d_{3}+d_{4}\right) e_{1}^{2}+d e_{1} / k_{*}$.

Now, we estimate $Z_{n+1}$ from $Z_{n}$. Using the partition of unity and Jensen's inequality, we get

$$
\begin{aligned}
Z_{n+1}= & \int_{\Omega \cap A_{k_{n+1}}}\left(u-k_{n+1}\right)^{\alpha(x)} \mathrm{d} x+\int_{\partial \Omega \cap A_{k_{n+1}}}\left(u-k_{n+1}\right)^{\beta(x)} \mathrm{d} S \\
= & \int_{\Omega \cap A_{k_{n+1}}}\left(u-k_{n+1}\right)^{\alpha(x)}\left(\sum_{i=1}^{m} \xi_{i}\right)^{\alpha_{+}} \mathrm{d} x \\
& +\int_{\partial \Omega \cap A_{k_{n+1}}}\left(u-k_{n+1}\right)^{\beta(x)}\left(\sum_{i=1}^{m} \xi_{i}\right)^{\beta_{+}} \mathrm{d} S
\end{aligned}
$$




$$
\begin{aligned}
& \leqslant m^{\alpha_{+}-1} \sum_{i=1}^{m} \int_{\Omega \cap A_{k_{n+1}}}\left(u-k_{n+1}\right)^{\alpha(x)} \xi_{i}^{\alpha+} \mathrm{d} x \\
& +m^{\beta_{+}-1} \sum_{i=1}^{m} \int_{\partial \Omega \cap A_{k_{n+1}}}\left(u-k_{n+1}\right)^{\beta(x)} \xi_{i}^{\beta_{+}} \mathrm{d} S \\
& \leqslant m^{\alpha_{+}-1} \sum_{i=1}^{m} \int_{\Omega \cap A_{k_{n+1}}}\left(u-k_{n+1}\right)^{\alpha(x)} \xi_{i}^{\alpha(x)} \mathrm{d} x \\
& +m^{\beta_{+}-1} \sum_{i=1}^{m} \int_{\partial \Omega \cap A_{k_{n+1}}}\left(u-k_{n+1}\right)^{\beta(x)} \xi_{i}^{\beta(x)} \mathrm{d} S .
\end{aligned}
$$

Thus

$$
\begin{aligned}
Z_{n+1} \leqslant m^{\max \left\{\alpha_{+}, \beta_{+}\right\}-1} \sum_{i=1}^{m}[ & \int_{\Omega \cap A_{k_{n+1}}}\left(u-k_{n+1}\right)^{\alpha_{+}} \xi_{i}^{\alpha_{+}} \mathrm{d} x \\
& +\int_{\partial \Omega \cap A_{k_{n+1}}}\left(u-k_{n+1}\right)^{\beta_{+}} \xi_{i}^{\beta_{+}} \mathrm{d} S \\
& +\int_{\Omega \cap A_{k_{n+1}}}\left(u-k_{n+1}\right)^{\alpha_{-}} \xi_{i}^{\alpha_{-}} \mathrm{d} x \\
& \left.+\int_{\partial \Omega \cap A_{k_{n+1}}}\left(u-k_{n+1}\right)^{\beta_{-}} \xi_{i}^{\beta_{-}} \mathrm{d} S\right] .
\end{aligned}
$$

For each $i \in\{1, \ldots, m\}$, define

$$
\begin{aligned}
& \tilde{\alpha}_{i}:= \begin{cases}\frac{1}{2}\left(\alpha_{i}^{+}+\left(p_{i}^{-}\right)^{*}\right) & \text { if }\left(p_{i}^{-}\right)^{*}<\infty, \\
\alpha_{i}^{+}+1 & \text { if }\left(p_{i}^{-}\right)^{*}=\infty,\end{cases} \\
& \tilde{\beta}_{i}:= \begin{cases}\frac{1}{2}\left(\beta_{i}^{+}+\left(p_{i}^{-}\right)^{\partial}\right) & \text { if }\left(p_{i}^{-}\right)^{\partial}<\infty, \\
\beta_{i}^{+}+1 & \text { if }\left(p_{i}^{-}\right)^{\partial}=\infty .\end{cases}
\end{aligned}
$$

Thus, $\alpha_{i}^{+}<\tilde{\alpha}_{i}<\left(p_{i}^{-}\right)^{*}$ and $\beta_{i}^{+}<\tilde{\beta}_{i}<\left(p_{i}^{-}\right)^{\partial}$, and so we deduce following continuous embedding:

$$
W^{1, p_{i}^{-}}(\Omega) \hookrightarrow L^{\tilde{\alpha}_{i}}(\Omega), \quad W^{1, p_{i}^{-}}(\Omega) \hookrightarrow L^{\tilde{\beta}_{i}}(\partial \Omega) .
$$

Let $i \in\{1, \ldots, m\}$ be fixed and suppose that $\gamma_{1} \in\left\{\alpha_{i}^{+}, \alpha_{i}^{-}\right\}, \gamma_{2} \in\left\{\beta_{i}^{+}, \beta_{i}^{-}\right\}$. Then, $p_{i}^{-} \leqslant \gamma_{1} \leqslant \alpha_{i}^{+}<\tilde{\alpha}_{i}<\left(p_{i}^{-}\right)^{*}$ and $p_{i}^{-} \leqslant \gamma_{2} \leqslant \beta_{i}^{+}<\tilde{\beta}_{i}<\left(p_{i}^{-}\right)^{\partial}$. Using the Hölder inequality and the claim about the embedding, we get

$$
\begin{aligned}
\int_{\Omega \cap A_{k_{n+1}}}\left(u-k_{n+1}\right)_{+}^{\gamma_{1}} \xi_{i}^{\gamma_{1}} \mathrm{~d} x+\int_{\partial \Omega \cap A_{k_{n+1}}}\left(u-k_{n+1}\right)_{+}^{\gamma_{2}} \xi_{i}^{\gamma_{2}} \mathrm{~d} S \\
\leqslant\left(\int_{\Omega}\left\{\xi_{i}\left(u-k_{n+1}\right)_{+}\right\}^{\tilde{\alpha}_{i}} \mathrm{~d} x\right)^{\gamma_{1} / \tilde{\alpha}_{i}}\left|\Omega \cap A_{k_{n+1}}\right|^{1-\gamma_{1} / \tilde{\alpha}_{i}} \\
\quad+\left(\int_{\partial \Omega}\left\{\xi_{i}\left(u-k_{n+1}\right)_{+}\right\}^{\tilde{\beta}_{i}} \mathrm{~d} S\right)^{\gamma_{2} / \tilde{\beta}_{i}}\left|\partial \Omega \cap A_{k_{n+1}}\right|^{1-\gamma_{2} / \tilde{\beta}_{i}}
\end{aligned}
$$




$$
\begin{aligned}
& \leqslant\left\|\xi_{i}\left(u-k_{n+1}\right)_{+}\right\|_{L^{\tilde{\alpha}_{i}(\Omega)}}^{\gamma_{1}}\left|A_{k_{n+1}}\right|^{1-\gamma_{1} / \tilde{\alpha}_{i}}+\left\|\xi_{i}\left(u-k_{n+1}\right)_{+}\right\|_{L^{\tilde{\beta}_{i}(\partial \Omega)}}^{\gamma_{2}}\left|A_{k_{n+1}}\right|^{1-\gamma_{2} / \tilde{\beta}_{i}} \\
& \leqslant C_{i}^{\gamma_{1}}\left(\left\|\xi_{i}\left(u-k_{n+1}\right)_{+}\right\|_{L^{p_{i}^{-}(\Omega)}}+\left\|\nabla\left\{\xi_{i}\left(u-k_{n+1}\right)_{+}\right\}\right\|_{L^{p_{i}^{-}}(\Omega)}\right)^{\gamma_{1}}\left|A_{k_{n+1}}\right|^{1-\gamma_{1} / \tilde{\alpha}_{i}} \\
& +D_{i}^{\gamma_{2}}\left(\left\|\xi_{i}\left(u-k_{n+1}\right)_{+}\right\|_{L^{p_{i}^{-}}(\Omega)}+\left\|\nabla\left\{\xi_{i}\left(u-k_{n+1}\right)_{+}\right\}\right\|_{L^{p_{i}^{-}}(\Omega)}\right)^{\gamma_{2}}\left|A_{k_{n+1}}\right|^{1-\gamma_{2} / \tilde{\beta}_{i}} \\
& \leqslant\left(2 C_{i}\right)^{\gamma_{1}}\left(\int_{\Omega}\left|\xi_{i}\left(u-k_{n+1}\right)_{+}\right|^{p_{i}^{-}} \mathrm{d} x\right. \\
& \left.+\int_{\Omega}\left|\nabla\left\{\xi_{i}\left(u-k_{n+1}\right)_{+}\right\}\right|^{p_{i}^{-}} \mathrm{d} x\right)^{\gamma_{1} / p_{i}^{-}}\left|A_{k_{n+1}}\right|^{1-\gamma_{1} / \tilde{\alpha}_{i}} \\
& +\left(2 D_{i}\right)^{\gamma_{2}}\left(\int_{\Omega}\left|\xi_{i}\left(u-k_{n+1}\right)_{+}\right|^{p_{i}^{-}} \mathrm{d} x\right. \\
& \left.+\int_{\Omega}\left|\nabla\left\{\xi_{i}\left(u-k_{n+1}\right)_{+}\right\}\right|^{p_{i}^{-}} \mathrm{d} x\right)^{\gamma_{2} / p_{i}^{-}}\left|A_{k_{n+1}}\right|^{1-\gamma_{2} / \tilde{\beta}_{i}},
\end{aligned}
$$

where $C_{i}$ and $D_{i}$ are the embedding constants. In addition, we have

$$
\int_{\Omega}\left|\xi_{i}\left(u-k_{n+1}\right)_{+}\right|^{p_{i}^{-}} \mathrm{d} x \leqslant \int_{\Omega \cap A_{k_{n+1}}} u^{\alpha(x)} \mathrm{d} x \leqslant e_{1}^{n+2} Z_{n},
$$

and from (4.3) and (4.7) we estimate

$$
\begin{aligned}
\int_{\Omega}\left|\nabla\left\{\xi_{i}\left(u-k_{n+1}\right)+\right\}\right|^{p_{i}^{-}} \mathrm{d} x & \leqslant 2^{p_{i}^{-}-1} \int_{\Omega \cap A_{k_{n+1}}}\left|\xi_{i} \nabla\left(u-k_{n+1}\right)\right|^{p_{i}^{-}} \mathrm{d} x \\
& +2^{p_{i}^{-}-1} \int_{\Omega \cap A_{k_{n+1}}}\left(u-k_{n+1}\right)^{p_{i}^{-}}\left|\nabla \xi_{i}\right|^{p_{i}^{-}} \mathrm{d} x \\
\leqslant & 2^{p_{i}^{-}-1} \frac{1}{d} e_{2} e_{1}^{n} Z_{n}+2^{p_{i}^{-}-1} L^{p_{i}^{-}} e_{1}^{n+2} Z_{n}
\end{aligned}
$$

where $L$ is a positive constant satisfying

$$
\left|\nabla \xi_{i}\right| \leqslant L \quad(i=1, \ldots, m)
$$

Using (4.10), relation (4.9) becomes

$$
\begin{aligned}
& \int_{\Omega \cap A_{k_{n+1}}}\left(u-k_{n+1}\right)_{+}^{\gamma_{1}} \xi_{i}^{\gamma_{1}} \mathrm{~d} x+\int_{\partial \Omega \cap A_{k_{n+1}}}\left(u-k_{n+1}\right)_{+}^{\gamma_{2}} \xi_{i}^{\gamma_{2}} \mathrm{~d} S \\
& \leqslant\left(2 C_{i}\right)^{\gamma_{1}}\left(e_{1}^{2}+2^{p_{i}^{-}-1} \frac{1}{d} e_{2}+2^{p_{i}^{-}-1} L^{p_{i}^{-}} e_{1}^{2}\right)^{\gamma_{1} / p_{i}^{-}}\left(e_{1}^{\gamma_{1} / p_{i}^{-}}\right)^{n} Z_{n}^{\gamma_{1} / p_{i}^{-}}\left|A_{k_{n+1}}\right|^{1-\gamma_{1} / \tilde{\alpha}_{i}} \\
& \quad+\left(2 D_{i}\right)^{\gamma_{2}}\left(e_{1}^{2}+2^{p_{i}^{-}-1} \frac{1}{d} e_{2}+2^{p_{i}^{-}-1} L^{p_{i}^{-}} e_{1}^{2}\right)^{\gamma_{2} / p_{i}^{-}}\left(e_{1}^{\gamma_{2} / p_{i}^{-}}\right)^{n} Z_{n}^{\gamma_{2} / p_{i}^{-}}\left|A_{k_{n+1}}\right|^{1-\gamma_{2} / \tilde{\beta}_{i}} .
\end{aligned}
$$

Noting that $Z_{n}^{R / p_{i}^{-}} \leqslant Z_{n}+Z_{n}^{\max \left\{\alpha_{+} / p_{-}, \beta_{+} / p_{-}\right\}}$for $R \in\left\{\gamma_{1}, \gamma_{2}\right\}$ and using (4.5), we have

$$
\left|A_{k_{n+1}}\right|^{1-\gamma_{1} / \tilde{\alpha}_{i}} \leqslant\left(\frac{e_{1}^{n+1}}{k_{*}} Z_{n}\right)^{1-\gamma_{1} / \tilde{\alpha}_{i}} \leqslant \frac{e_{1}^{1-\gamma_{1} / \tilde{\alpha}_{i}}}{k_{*}^{1-\eta}}\left(e_{1}^{1-\gamma_{1} / \tilde{\alpha}_{i}}\right)^{n}\left(Z_{n}+Z_{n}^{1-\eta}\right)
$$


and

$$
\left|A_{k_{n+1}}\right|^{1-\gamma_{1} / \tilde{\beta}_{i}} \leqslant\left(\frac{e_{1}^{n+1}}{k_{*}} Z_{n}\right)^{1-\gamma_{2} / \tilde{\beta}_{i}} \leqslant \frac{e_{1}^{1-\gamma_{1} / \tilde{\beta}_{i}}}{k_{*}^{1-\eta}}\left(e_{1}^{1-\gamma_{2} / \tilde{\beta}_{i}}\right)^{n}\left(Z_{n}+Z_{n}^{1-\eta}\right),
$$

where $\eta:=\max _{1 \leqslant i \leqslant m}\left\{\alpha_{i}^{+} / \tilde{\alpha}_{i}, \beta_{i}^{+} / \tilde{\beta}_{i}\right\}<1$. It follows from (4.11)-(4.13) that

$$
\begin{aligned}
& \int_{\Omega \cap A_{k_{n+1}}}\left(u-k_{n+1}\right)^{\gamma_{1}} \xi_{i}^{\gamma_{1}} \mathrm{~d} x+\int_{\partial \Omega \cap A_{k_{n+1}}}\left(u-k_{n+1}\right)^{\gamma_{2}} \xi_{i}^{\gamma_{2}} \mathrm{~d} S \\
& \leqslant \frac{e_{\gamma_{1}}}{k_{*}^{1-\eta}}\left(e_{1}^{\gamma_{1} / p_{i}^{-}}\right)^{n}\left(e_{1}^{1-\gamma_{1} / \tilde{\alpha}_{i}}\right)^{n}\left(Z_{n}+Z_{n}^{1-\eta}\right)\left(Z_{n}+Z_{n}^{\max \left\{\alpha_{+} / p_{-}, \beta_{+} / p_{-}\right\}}\right) \\
& +\frac{e_{\gamma_{2}}}{k_{*}^{1-\eta}}\left(e_{1}^{\gamma_{2} / p_{i}^{-}}\right)^{n}\left(e_{1}^{1-\gamma_{2} / \tilde{\beta}_{i}}\right)^{n}\left(Z_{n}+Z_{n}^{1-\eta}\right)\left(Z_{n}+Z_{n}^{\max \left\{\alpha_{+} / p_{-}, \beta_{+} / p_{-}\right\}}\right) \\
& \leqslant\left(e_{\gamma_{1}}+e_{\gamma_{2}}\right) \frac{1}{k_{*}^{1-\eta}}\left(e_{1}^{1+\left(\alpha_{+}+\beta_{+}\right) / p^{-}}\right)^{n}\left(Z_{n}+Z_{n}^{1-\eta}\right)\left(Z_{n}+Z_{n}^{\max \left\{\alpha_{+} / p_{-}, \beta_{+} / p_{-}\right\}}\right),
\end{aligned}
$$

where

$$
e_{\gamma_{1}}:=\left(2 C_{i}\right)^{\gamma_{1}}\left(e_{1}^{2}+2^{p_{i}^{-}-1} d^{-1} e_{2}+2^{p_{i}^{-}-1} L^{p_{i}^{-}} e_{1}^{2}\right)^{\gamma_{1} / p_{i}^{-}} e_{1}^{1-\gamma_{1} / \tilde{\alpha}_{i}}
$$

and

$$
e_{\gamma_{2}}:=\left(2 D_{i}\right)^{\gamma_{2}}\left(e_{1}^{2}+2^{p_{i}^{-}-1} d^{-1} e_{2}+2^{p_{i}^{-}-1} L^{p_{i}^{-}} e_{1}^{2}\right)^{\gamma_{2} / p_{i}^{-}} e_{1}^{1-\gamma_{2} / \tilde{\beta}_{i}}
$$

We deduce from (4.8) and (4.14) that

$$
Z_{n+1} \leqslant \frac{e_{3}}{4 k_{*}^{1-\eta}} b^{n}\left(Z_{n}^{2}+Z_{n}^{2-\eta}+Z_{n}^{1+\max \left\{\alpha_{+} / p_{-}, \beta_{+} / p_{-}\right\}}+Z_{n}^{1+\max \left\{\alpha_{+} / p_{-}, \beta_{+} / p_{-}\right\}-\eta}\right),
$$

where

$$
\begin{gathered}
e_{3}=4 m^{\max \left\{\alpha_{+}, \beta_{+}\right\}-1} \sum_{i=1}^{m}\left(e_{\alpha_{i}^{+}}+e_{\beta_{i}^{+}}+e_{\alpha_{i}^{-}}+e_{\beta_{i}^{-}}\right)>0 \\
b=e_{1}^{1+\left(\alpha_{+}+\beta_{+}\right) / p_{-}}>1 .
\end{gathered}
$$

Arguing the cases $Z_{n} \geqslant 1$ and $Z_{n}<1$, and noting that the smallest and the largest exponents are $2-\eta$ and $1+\max \left\{\alpha_{+} / p_{-}, \beta_{+} / p_{-}\right\}$, respectively, we get

$$
Z_{n+1} \leqslant \frac{e_{3}}{k_{*}^{1-\eta}} b^{n}\left(Z_{n}^{2-\eta}+Z_{n}^{1+\max \left\{\alpha_{+} / p_{-}, \beta_{+} / p_{-}\right\}}\right) .
$$

In other words,

$$
Z_{n+1} \leqslant \frac{e_{3}}{k_{*}^{\delta_{1}}} b^{n}\left(Z_{n}^{1+\delta_{1}}+Z_{n}^{1+\delta_{2}}\right)
$$

where $0<\delta_{1}=1-\eta<1 \leqslant \delta_{2}=\max \left\{\alpha_{+} / p_{-}, \beta_{+} / p_{-}\right\}$. Applying lemma 4.2 with (4.15), we obtain that

$$
Z_{n}=\int_{\Omega}\left(u-k_{n}\right)_{+}^{\alpha(x)} \mathrm{d} x+\int_{\partial \Omega}\left(u-k_{n}\right)_{+}^{\beta(x)} \mathrm{d} S \rightarrow 0 \quad \text { as } n \rightarrow \infty
$$


provided that

$$
Z_{0} \leqslant \min \left\{1, k_{*}^{\delta_{1}-1 / \delta_{1}} b^{-1 / \delta_{1}^{2}}\right\}
$$

or

$$
Z_{0} \leqslant \min \left\{\left(\frac{2 e_{3}}{k_{*}^{\delta_{1}}}\right)^{-1 / \delta_{1}} b^{-1 / \delta_{1}^{2}},\left(\frac{2 e_{3}}{k_{*}^{\delta_{1}}}\right)^{-1 / \delta_{2}} b^{-1 /\left(\delta_{1} \delta_{2}\right)-\left(\delta_{2}-\delta_{1}\right) / \delta_{2}^{2}}\right\}
$$

Observe that

$$
\begin{aligned}
Z_{0} & =\int_{\Omega \cap A_{k_{*}}}\left(u-k_{*}\right)^{\alpha(x)} \mathrm{d} x+\int_{\partial \Omega \cap A_{k_{*}}}\left(u-k_{*}\right)^{\beta(x)} \mathrm{d} S \\
& \leqslant \int_{\Omega} u_{+}^{\alpha(x)} \mathrm{d} x+\int_{\partial \Omega} u_{+}^{\beta(x)} \mathrm{d} S .
\end{aligned}
$$

Set

$$
k_{*}=\left[1+\left(2 e_{3}\right)^{1 / \delta_{1}} b^{1 / \delta_{1}^{2}+\left(\delta_{2}-\delta_{1}\right) / \delta_{1} \delta_{2}}\right]\left[1+\left(\int_{\Omega} u_{+}^{\alpha(x)} \mathrm{d} x+\int_{\partial \Omega} u_{+}^{\beta(x)} \mathrm{d} S\right)^{\delta_{2} / \delta_{1}}\right] .
$$

So we have inequality (4.17) if $\rho \leqslant 1$ or inequality (4.18) if $\rho>1$, where

$$
\rho=\int_{\Omega} u_{+}^{\alpha(x)} \mathrm{d} x+\int_{\partial \Omega} u_{+}^{\beta(x)} \mathrm{d} S .
$$

Since $k_{n} \uparrow 2 k_{*},(4.16)$ implies that

$$
\int_{\Omega}\left(u-2 k_{*}\right)_{+}^{\alpha(x)} \mathrm{d} x+\int_{\partial \Omega}\left(u-2 k_{*}\right)_{+}^{\beta(x)} \mathrm{d} S=0 .
$$

Consequently, $\left(u-2 k_{*}\right)_{+}=0$ a.e. in $\bar{\Omega}$ and this means that $\operatorname{ess} \sup _{\bar{\Omega}} u \leqslant 2 k_{*}$. The boundedness from below of $u$ can be shown analogously by replacing $u$ with $-u$, $A_{k}$ with $\tilde{A}_{k}$, and using (4.2) instead of (4.1). This completes the proof.

The following lemma is quoted from [19].

Lemma 4.4 (Heinz [19]). Let $I \in C^{1}(X, \mathbb{R})$, where $X$ is a Banach space. Assume that $I$ satisfies the (PS)-condition, is even and bounded from below, and $I(0)=0$. If for any $n \in \mathbb{N}$ there exists an $n$-dimensional subspace $X_{n}$ and a $\rho_{n}>0$ such that

$$
\sup _{X_{n} \cap S_{\rho_{n}}} I<0
$$

where $S_{\rho}:=\left\{u \in X:\|u\|_{X}=\rho\right\}$, then $I$ has a sequence of critical values $c_{n}<0$ satisfying $c_{n} \rightarrow 0$ as $n \rightarrow \infty$.

Lemma 4.5. Assume that (J1), (J2), (J4), (J6), (F1), (F2) and (G1) hold. If, furthermore,

$$
p_{-} F(x, t)-f(x, t) t>0 \quad \text { for all } x \in \Omega \text { and for } t \neq 0
$$

and

$$
p_{-} G(x, t)-g(x, t) t>0 \quad \text { for all } x \in \partial \Omega \text { and for } t \neq 0
$$


then

$$
I(u)=\left\langle I^{\prime}(u), u\right\rangle=0 \quad \text { if and only if } u=0 .
$$

Proof. Let $I(u)=\left\langle I^{\prime}(u), u\right\rangle=0$. Then we see that

$$
\begin{aligned}
0=-p_{-} I(u)=- & p_{-} \int_{\Omega} \Phi_{0}(x, \nabla u) \mathrm{d} x-p_{-} \int_{\Omega} \frac{1}{p(x)}|u|^{p(x)} \mathrm{d} x \\
& +p_{-} \int_{\Omega} F(x, u) \mathrm{d} x+p_{-} \int_{\partial \Omega} G(x, u) \mathrm{d} S \\
\geqslant- & p_{-} \int_{\Omega} \Phi_{0}(x, \nabla u) \mathrm{d} x-\int_{\Omega}|u|^{p(x)} \mathrm{d} x \\
& +p_{-} \int_{\Omega} F(x, u) \mathrm{d} x+p_{-} \int_{\partial \Omega} G(x, u) \mathrm{d} S,
\end{aligned}
$$

and

$$
\begin{aligned}
\left\langle I^{\prime}(u), u\right\rangle= & \int_{\Omega} \varphi(x, \nabla u) \cdot \nabla u \mathrm{~d} x+\int_{\Omega}|u|^{p(x)} \mathrm{d} x \\
& \quad-\int_{\Omega} f(x, u) u \mathrm{~d} x-\int_{\partial \Omega} g(x, u) u \mathrm{~d} S \\
= & 0 .
\end{aligned}
$$

It follows from assumption (J6) and relations (4.21) and (4.22) that

$$
\int_{\Omega}\left\{p_{-} F(x, u)-f(x, u) u\right\} \mathrm{d} x+\int_{\partial \Omega}\left\{p_{-} G(x, u)-g(x, u) u\right\} \mathrm{d} S \leqslant 0 .
$$

Consequently, assumptions (4.19) and (4.20) imply that $u=0$. The converse is clear from assumption (J1).

Lemma 4.6. Assume that (F1), (F2), (F6), (F7), (G1), (G5) and (G6) hold. Then there are $t_{0}>0, \tilde{f} \in C^{1}(\Omega \times \mathbb{R}, \mathbb{R})$ and $\tilde{g} \in C^{1}(\partial \Omega \times \mathbb{R}, \mathbb{R})$ such that $\tilde{f}(x, t)$ and $\tilde{g}(x, t)$ are odd in $t$ and satisfy

$$
\begin{gathered}
\tilde{\mathcal{F}}(x, t)=p_{-} \tilde{F}(x, t)-\tilde{f}(x, t) t \geqslant 0, \\
\tilde{\mathcal{G}}(x, t)=p_{-} \tilde{G}(x, t)-\tilde{g}(x, t) t \geqslant 0, \\
\tilde{\mathcal{F}}(x, t)=0 \quad \Longleftrightarrow \quad t \equiv 0 \text { or }|t| \geqslant 2 t_{0}, \\
\tilde{\mathcal{G}}(x, t)=0 \quad \Longleftrightarrow \quad t \equiv 0 \text { or }|t| \geqslant 2 t_{0} .
\end{gathered}
$$

Proof. Let us define a cut-off function $\kappa_{1} \in C^{1}(\mathbb{R}, \mathbb{R})$ satisfying $\kappa_{1}(t)=1$ for $|t| \leqslant t_{0}, \kappa_{1}(t)=0$ for $|t| \geqslant 2 t_{0},\left|\kappa_{1}^{\prime}(t)\right| \leqslant 2 / t_{0}$ and $\kappa_{1}^{\prime}(t) t \leqslant 0$. So, we define

$$
\tilde{F}(x, t)=\kappa_{1}(t) F(x, t)+\left(1-\kappa_{1}(t)\right) \gamma_{1}|t|^{p_{-}} \quad \text { and } \quad \tilde{f}(x, t)=\frac{\partial}{\partial t} \tilde{F}(x, t),
$$

where $\gamma_{1}>0$ is a constant. It is straightforward to see that

$$
p_{-} \tilde{F}(x, t)-\tilde{f}(x, t) t=\kappa_{1}(t) \mathcal{F}(x, t)-\kappa_{1}^{\prime}(t) t F(x, t)+\kappa_{1}^{\prime}(t) t \gamma_{1}|t|^{p_{-}},
$$


where $\mathcal{F}(x, t):=p_{-} F(x, t)-f(x, t) t$. For $0 \leqslant|t| \leqslant t_{0}$ and $|t| \geqslant 2 t_{0}$ the conclusion follows. By (F7), we choose a sufficiently small $t_{0}>0$ such that $F(x, t) \geqslant \gamma_{1} t^{p_{-}}$for $t_{0} \leqslant|t| \leqslant 2 t_{0}$. Due to the assumption that $\kappa_{1}^{\prime}(t) t \leqslant 0$ we get the conclusion.

Let us define a cut-off function $\kappa_{2} \in C^{1}(\mathbb{R}, \mathbb{R})$ satisfying $\kappa_{2}(t)=1$ for $|t| \leqslant t_{0}$, $\kappa_{2}(t)=0$ for $|t| \geqslant 2 t_{0},\left|\kappa_{2}^{\prime}(t)\right| \leqslant 2 / t_{0}$ and $\kappa_{2}^{\prime}(t) t \leqslant 0$. As in (4.23), we define

$$
\tilde{G}(x, t)=\kappa_{2}(t) G(x, t)+\left(1-\kappa_{2}(t)\right) \gamma_{2}|t|^{p_{-}} \quad \text { and } \quad \tilde{g}(x, t)=\frac{\partial}{\partial t} \tilde{G}(x, t)
$$

where $\gamma_{2}>0$ is a constant. From analogous arguments, we deduce the conclusion.

Now we prove the second main result using proposition 4.3 and lemmas 4.4 and 4.6.

THEOREM 4.7. Assume that (J1)-(J4), (J6), (F1), (F2), (F5)-(F7), (G1) and (G4)-(G6) hold. If $\Phi_{0}(x,-v)=\Phi_{0}(x, v)$ holds for all $(x, v) \in \Omega \times \mathbb{R}^{N}$, then problem $(P)$ has a sequence of weak solutions $u_{n}$ such that $\left\|u_{n}\right\|_{L^{\infty}(\bar{\Omega})} \rightarrow 0$ as $n \rightarrow \infty$.

Proof. Let $u \in X$ and $\|u\|_{X}>1$. We can choose positive constants $c_{1}$ and $c_{2}$ satisfying

$$
\|u\|_{L^{p_{-}(\Omega)}}^{p_{-}} \leqslant c_{1}\|u\|_{X}^{p_{-}} \quad \text { and } \quad\|u\|_{L^{p_{-}(\partial \Omega)}}^{p_{-}} \leqslant c_{2}\|u\|_{X}^{p_{-}}
$$

For the given functions $f(x, t)$ and $g(x, t)$, we can modify and extend $\tilde{f} \in C^{1}(\Omega \times$ $\mathbb{R}, \mathbb{R})$ and $\tilde{g} \in C^{1}(\partial \Omega \times \mathbb{R}, \mathbb{R})$ satisfying all properties listed in lemma 4.6 with $\gamma_{1}$ and $\gamma_{2}$ such that $p_{+}\left(c_{1} \gamma_{1}+c_{2} \gamma_{2}\right) \leqslant \min \{d, 1\}$ for the positive constant $d$ from $(\mathrm{J} 4)$. And also, by lemma 4.6 , it is easy to show that $\tilde{I} \in C^{1}(X, \mathbb{R})$ and is even on $X$. Moreover,

$$
\begin{aligned}
\tilde{I}(u):= & \int_{\Omega} \Phi_{0}(x, \nabla u) \mathrm{d} x+\int_{\Omega} \frac{1}{p(x)}|u|^{p(x)} \mathrm{d} x-\int_{\Omega} \tilde{F}(x, u) \mathrm{d} x-\int_{\partial \Omega} \tilde{G}(x, u) \mathrm{d} S \\
\geqslant & \frac{d}{p_{+}} \int_{\Omega}|\nabla u|^{p(x)} \mathrm{d} x+\frac{1}{p_{+}} \int_{\Omega}|u|^{p(x)} \mathrm{d} x \\
& -\int_{\Omega \cap \Omega_{1}}\left\{\kappa_{1}(u) F(x, u)+\left(1-\kappa_{1}(u)\right) \gamma_{1}|u|^{p_{-}}\right\} \mathrm{d} x-\int_{\Omega \cap \Omega_{2}} \gamma_{1}|u|^{p_{-}} \mathrm{d} x \\
& -\int_{\partial \Omega \cap \Omega_{1}}\left\{\kappa_{2}(u) G(x, u)+\left(1-\kappa_{2}(u)\right) \gamma_{2}|u|^{p_{-}}\right\} \mathrm{d} S-\int_{\partial \Omega \cap \Omega_{2}} \gamma_{2}|u|^{p_{-}} \mathrm{d} S \\
\geqslant & \frac{\min \{d, 1\}}{p_{+}}\|u\|_{X}^{p_{-}}-\int_{\Omega \cap \Omega_{1}} F(x, u) \mathrm{d} x-\int_{\Omega} \gamma_{1}|u|^{p_{-}} \mathrm{d} x-\int_{\partial \Omega \cap \Omega_{1}} G(x, u) \mathrm{d} S \\
& -\int_{\partial \Omega} \gamma_{2}|u|^{p_{-}} \mathrm{d} S \\
\geqslant & \frac{\min \{d, 1\}}{p_{+}}\|u\|_{X}^{p_{-}}-\gamma_{1} c_{1}\|u\|_{X}^{p_{-}}-\gamma_{2} c_{2}\|u\|_{X}^{p_{-}} \\
& -\int_{\Omega \cap \Omega_{1}}\left\{d_{1}|u|+\frac{d_{2}}{p_{+}}|u|^{\alpha(x)}\right\} \mathrm{d} x-\int_{\partial \Omega \cap \Omega_{1}}\left\{d_{3}|u|+\frac{d_{4}}{p_{+}}|u|^{\beta(x)}\right\} \mathrm{d} S,
\end{aligned}
$$


where $\Omega_{1}:=\left\{x \in \bar{\Omega}: 0 \leqslant|u(x)| \leqslant 2 t_{0}\right\}$ and $\Omega_{2}:=\left\{x \in \bar{\Omega}: 2 t_{0} \leqslant|u(x)|\right\}$. Thus, we have

$$
\tilde{I}(u) \geqslant \frac{\min \{d, 1\}}{p_{+}}\|u\|_{X}^{p_{-}}-\left(\gamma_{1} c_{1}+\gamma_{2} c_{2}\right)\|u\|_{X}^{p_{-}}+C_{10}
$$

for some positive constant $C_{10}$, so that $\tilde{I}$ is coercive, that is, $\tilde{I}(u) \rightarrow \infty$ as $\|u\|_{X} \rightarrow$ $\infty$. By a standard argument, $\tilde{I}$ satisfies the $(\mathrm{PS})_{c}$-condition. In order to apply lemma 4.4, we only need to find, for any $n \in \mathbb{N}$, a subspace $X_{n}$ and a $\rho_{n}>0$ such that $\sup _{X_{n} \cap S_{\rho_{n}}} \tilde{I}<0$. For any $n \in \mathbb{N}$ we find $n$ independent smooth functions $\phi_{i}$ for $i=1, \ldots, n$, and define $X_{n}:=\operatorname{span}\left\{\phi_{1}, \ldots, \phi_{n}\right\}$. By (J2) and lemma 4.6, when $\|u\|_{X}<1$ we have that

$$
\begin{aligned}
\tilde{I}(u) & :=\int_{\Omega} \Phi_{0}(x, \nabla u) \mathrm{d} x+\int_{\Omega} \frac{1}{p(x)}|u|^{p(x)} \mathrm{d} x-\int_{\Omega} \tilde{F}(x, u) \mathrm{d} x-\int_{\partial \Omega} \tilde{G}(x, u) \mathrm{d} S \\
& \leqslant\left(2\|a\|_{L^{p^{\prime}(\cdot)}(\Omega)}+b+1\right)\|u\|_{X}^{p_{-}}-C_{11} \int_{\Omega} F(x, u) \mathrm{d} x-C_{11} \int_{\partial \Omega} G(x, u) \mathrm{d} S
\end{aligned}
$$

for a positive constant $C_{11}$. It follows from assumptions (F7) and (G6) that, for a sufficiently large $M_{0}>0$, there exists $\delta_{0}>0$ such that $|t|<\delta_{0}$ implies both

$$
\int_{\Omega} F(x, t) \mathrm{d} x \geqslant \frac{M_{0}}{p_{-}} \int_{\Omega}|t|^{p_{-}} \mathrm{d} x \quad \text { and } \quad \int_{\partial \Omega} G(x, t) \mathrm{d} S \geqslant \frac{M_{0}}{p_{-}} \int_{\partial \Omega}|t|^{p_{-}} \mathrm{d} S .
$$

By this and the fact that all norms on $X_{n}$ are equivalent, choosing a suitable constant $C_{11}$ and sufficiently small $\rho_{n}>0$, we can obtain

$$
\sup _{X_{n} \cap S_{\rho_{n}}} \tilde{I}<0
$$

By lemma 4.4, we get a sequence $c_{n}<0$ for $\tilde{I}$ satisfying $c_{n} \rightarrow 0$ when $n$ goes to $\infty$. Then for any $u_{n} \in X$ satisfying $\tilde{I}\left(u_{n}\right)=c_{n}$ and $\tilde{I}^{\prime}\left(u_{n}\right)=0$, the sequence $\left\{u_{n}\right\}$ is a $(\mathrm{PS})_{0}$-sequence of $\tilde{I}(u)$, and $\left\{u_{n}\right\}$ has a convergent subsequence. By lemmas 4.5 and $4.6, \mathbf{0}$ is the only critical point with 0 energy and the subsequence of $\left\{u_{n}\right\}$ has to converge to $\mathbf{0}$. An indirect argument shows that the sequence $\left\{u_{n}\right\}$ has to converge to $\mathbf{0}$. On the other hand, by proposition $4.3, u_{n} \in C(\bar{\Omega})$. Since $\left\|u_{n}\right\|_{L^{\infty}(\bar{\Omega})} \rightarrow 0$, by lemma 4.6 again, we have $\left\|u_{n}\right\|_{C(\bar{\Omega})} \leqslant t_{0}$. Thus, $\left\{u_{n}\right\}$ are weak solutions of problem (P). The proof is complete.

\section{Acknowledgements}

J.-M.K. was partly supported by BK21 PLUS SNU Mathematical Sciences Division and Y.-H.K. was supported by the Basic Science Research Program through the National Research Foundation of Korea (NRF) funded by the Ministry of Education (Grant no. NRF-2014R1A1A2059536).

\section{References}

1 A. Ambrosetti and P. Rabinowitz. Dual variational methods in critical point theory and applications. J. Funct. Analysis 14 (1973), 349-381.

2 G. Barletta and A. Chinnì. Existence of solutions for a Neumann problem involving the $p(x)$-Laplacian. Electron. J. Diff. Eqns 158 (2013), 1-12. 
3 G. Barletta, A. Chinnì and D. O'Regan. Existence results for a Neumann problem involving the $p(x)$-Laplacian with discontinuous nonlinearities. Nonlin. Analysis 27 (2016), 312-325.

4 G. Bin. On superlinear $p(x)$-Laplacian-like problem without Ambrosetti and Rabinowitz condition. Bull. Korean Math. Soc. 51 (2014), 409-421.

5 G. Bonanno. A critical point theorem via the Ekeland variational principle. Nonlin. Analysis 75 (2012), 2992-3007.

6 M.-M. Boureanu and F. Preda. Infinitely many solutions for elliptic problems with variable exponent and nonlinear boundary conditions. Nonlin. Diff. Eqns Applic. 19 (2012), 235251.

7 E. B. Choi and Y.-H. Kim. Existence of nontrivial solutions for equations of $p(x)$-Laplace type without Ambrosetti and Rabinowitz condition. In Dynamical systems, differential equations and applications: discrete and continuous dynamical systems - proceedings 2015, pp. 276-286 (Springfield, MO: American Institute of Mathematical Sciences, 2015).

8 N. T. Chung. Multiple solutions for quasilinear elliptic problems with nonlinear boundary conditions. Electron. J. Diff. Eqns 2008 (2008), 1-6.

9 N. T. Chung and H. Q. Toan. On a nonlinear and non-homogeneous problem without (A-R) type condition in Orlicz-Sobolev spaces. Appl. Math. Computat. 219 (2013), 7820-7829.

10 L. Diening, P. Harjulehto, P. Hästö and M. Rúžička. Lebesgue and Sobolev Spaces with variable exponents, Lecture Notes in Mathematics, vol. 2017 (Springer, 2011).

11 D. E. Edmunds and J. Rákosník. Sobolev embedding with variable exponent. Studia Math. 143 (2000), 267-293.

12 X. Fan. Solutions for $p(x)$-Laplacian Dirichlet problems with singular coefficients. J. Math. Analysis Applic. 312 (2005), 464-477.

13 X. Fan. Global $C^{1, \alpha}$ regularity for variable exponent elliptic equations in divergence form. J. Diff. Eqns 235 (2007), 397-417.

14 X. Fan. Boundary trace embedding theorems for variable exponent Sobolev spaces. J. Math. Analysis Applic. 339 (2008), 1395-1412.

15 X. Fan and Q. H. Zhang. Existence of solutions for $p(x)$-Laplacian Dirichlet problem. Nonlin. Analysis 52 (2003), 1843-1852.

16 X. Fan and D. Zhao. On the spaces $L^{p(x)}(\Omega)$ and $W^{m, p(x)}(\Omega)$. J. Math. Analysis Applic. 263 (2001), 424-446.

17 H. Galewski. On the continuity of the Nemyskij operator between the spaces $L^{p_{1}(x)}$ and $L^{p_{2}(x)}$. Georgian Math. J. 13 (2006), 261-265.

18 B. Ge. On the superlinear problems involving the $p(x)$-Laplacian and a non-local term without AR-condition. Nonlin. Analysis 102 (2014), 133-143.

19 H. P. Heinz. Free Ljusternik-Schnirelman theory and the bifurcation diagrams of certain singular nonlinear problems. J. Diff. Eqns 66 (1987), 263-300.

20 K. Ho and I. Sim. Existence and some properties of solutions for degenerate elliptic equations with exponent variable. Nonlin. Analysis 98 (2014), 146-164.

21 K. Ho and I. Sim. Corrigendum to 'Existence and some properties of solutions for degenerate elliptic equations with exponent variable'. Nonlin. Analysis 128 (2015), 423-426.

22 C. Ji. On the superlinear problem involving the $p(x)$-Laplacian. Electron. J. Qual. Theory Differ. 40 (2011), 1-9.

23 I. H. Kim and Y.-H. Kim. Mountain pass type solutions and positivity of the infimum eigenvalue for quasilinear elliptic equations with variable exponents. Manuscr. Math. $\mathbf{1 4 7}$ (2015), 169-191.

24 V. K. Le. On a sub-supersolution method for variational inequalities with Leray-Lions operators in variable exponent spaces. Nonlin. Analysis 71 (2009), 3305-3321.

25 S. D. Lee, K. Park and Y.-H. Kim. Existence and multiplicity of solutions for equations involving nonhomogeneous operators of $p(x)$-Laplace type in $\mathbb{R}^{N}$. Bound. Value Probl. 2014 (2014), 1-17.

26 G. Li and C. Yang. The existence of a nontrivial solution to a nonlinear elliptic boundary value problem of $p$-Laplacian type without the Ambrosetti-Rabinowitz condition. Nonlin. Analysis 72 (2010), 4602-4613.

27 Q. Liu and D. Liu. Existence and multiplicity of solutions to a $p(x)$-Laplacian equation with nonlinear boundary condition on unbounded domain. Diff. Eqns Applic. 5 (2013), 595-611. 
F.-Y. Lu and G.-Q. Deng. Infinitely many weak solutions of the $p$-Laplacian equation with nonlinear boundary conditions. Sci. World J. 2014 (2014), 1-5.

29 M. Mihăilescu and V. Rădulescu. A multiplicity result for a nonlinear degenerate problem arising in the theory of electrorheological fluids. Proc. R. Soc. Edinb. A 462 (2006), 26252641.

30 O. H. Miyagaki and M. A. S. Souto. Superlinear problems without Ambrosetti and Rabinowitz growth condition. J. Diff. Eqns 245 (2008), 3628-3638.

31 D. Naimen. Existence of infinitely many solutions for nonlinear Neumann problems with indefinite coefficients. Electron. J. Diff. Eqns 2014 (2014), 1-12.

32 P. Pucci and Q. Zhang. Existence of entire solutions for a class of variable exponent elliptic equations. J. Diff. Eqns 257 (2014), 1529-1566.

33 M. Rǚ̌ička. Electrorheological fluids: modeling and mathematical theory, Lecture Notes in Mathematics, vol. 1748 (Springer, 2000).

34 Z. Tan and F. Fang. On superlinear $p(x)$-Laplacian problems without Ambrosetti and Rabinowitz condition. Nonlin. Analysis 75 (2012), 3902-3915.

35 V. Vergara and R. Zacher. A priori bounds for degenerate and singular evolutionary partial integro-differential equations. Nonlin. Analysis $\mathbf{7 3}$ (2010), 3572-3585.

36 Z.-Q. Wang. Nonlinear boundary value problems with concave nonlinearities near the origin. Nonlin. Diff. Eqns Applic. 8 (2001), 15-33.

37 M. Willem. Minimax theorems (Birkhäuser, 1996).

38 P. Winkert. Multiplicity results for a class of elliptic problems with nonlinear boundary condition. Commun. Pure Appl. Analysis 12 (2013), 785-802.

39 P. Winkert and R. Zacher. A priori bounds for weak solutions to elliptic equations with nonstandard growth. Discrete Contin. Dynam. Syst. S 5 (2012), 865-878.

40 J. Yao. Solutions for Neumann boundary value problems involving $p(x)$-Laplace operators. Nonlin. Analysis 68 (2008), 1271-1283.

41 J.-H. Zhao and P.-H. Zhao. Existence of infinitely many weak solutions for the $p$-Laplacian with nonlinear boundary conditions. Nonlin. Analysis 69 (2008), 1343-1355. 